\title{
Isotope dependence of the type I ELMy H-mode pedestal in JET-ILW Hydrogen and Deuterium plasmas
}

L. Horvath ${ }^{1}$, C.F. Maggi ${ }^{2}$, A. Chankin ${ }^{3}$, S. Saarelma ${ }^{2}$, A.R. Field $^{2}$, S. Aleiferis ${ }^{4}$, E. Belonohy ${ }^{2}$, A. Boboc ${ }^{2}$, G. Corrigan ${ }^{2}$, E.G. Delabie ${ }^{5}$, J. Flanagan ${ }^{2}$, L. Frassinetti ${ }^{6}$, C. Giroud ${ }^{2}$, D. Harting $^{2}$, D. Keeling ${ }^{2}$, D. King ${ }^{2}$, M. Maslov ${ }^{2}$, G.F. Matthews ${ }^{2}$, S. Menmuir ${ }^{2}$, S.A. Silburn ${ }^{2}$, J. Simpson ${ }^{2,7}$, A.C.C Sips ${ }^{8,9}$, H. Weisen $^{10}$, K.J. Gibson ${ }^{1}$ and JET Contributors*

EUROfusion Consortium, JET, Culham Science Centre, Abingdon OX14 3DB, UK

${ }^{1}$ York Plasma Institute, Department of Physics, University of York, York, YO10 5DD, UK

${ }^{2}$ CCFE, Culham Science Centre, Abingdon, OX14 3DB, UK

${ }^{3}$ Max-Planck-Institut für Plasmaphysik, Boltzmannstrasse 2, D-85748, Garching, Germany

${ }^{4}$ Fusion Technology Group, National Centre for Scientific Research "Demokritos", 15310 Aghia Paraskevi, Athens, Greece

${ }^{5}$ Oak Ridge National Laboratory, Oak Ridge, Tennessee, United States of America

${ }^{6}$ Division of Fusion Plasma Physics, KTH Royal Institute of Technology,

Stockholm, Sweden

${ }^{7}$ Aalto University, 02150 Espoo, Finland

${ }^{8}$ JET Exploitation Unit, Culham Science Centre, Abingdon, OX14 3DB, UK

${ }^{9}$ European Commission, Brussels, Belgium

${ }^{1}$ 0SPC, Ecole Polytechnique Federale de Lausanne, Switzerland

* See the author list of E. Joffrin et al. accepted for publication in Nuclear Fusion

Special issue 2019, https://doi.org/10.1088/1741-4326/ab2276

E-mail: laszlo.horvath@ukaea.uk

\begin{abstract}
.
The pedestal structure, edge transport and linear MHD stability are analysed in a series of JET-ILW Hydrogen (H) and Deuterium (D) type I ELMy H-mode plasmas. The pedestal pressure is typically higher in $\mathrm{D}$ than in $\mathrm{H}$ at the same input power and gas rate, with the difference mainly due to lower density in $H$ than in $\mathrm{D}$ [1]. The neutral penetration model [2] alone does not explain the difference in edge density profile between $\mathrm{H}$ and $\mathrm{D}$. It is in contradiction with the narrower (or similar) pedestal density width in $\mathrm{H}$ than in $\mathrm{D}$ implying that different edge transport must also play a role. Interpretative EDGE2D-EIRENE simulations confirm that the difference in neutral penetration between $\mathrm{H}$ and $\mathrm{D}$ leads only to minor changes in the upstream density and temperature profiles and with trends opposite to experimental observations. Higher edge particle and heat transport coefficients are needed in $\mathrm{H}$ than in $\mathrm{D}$ to match the experimental profiles with EDGE2D-EIRENE, suggesting that the higher edge transport in $\mathrm{H}$ than in $\mathrm{D}$ is the main reason for the different pedestals. Higher
\end{abstract}


inter-ELM separatrix loss power is required in $\mathrm{H}$ than in $\mathrm{D}$ to maintain a similar pedestal top pressure. The ELM energy losses are dominated by particle losses both in $\mathrm{H}$ and $\mathrm{D}$ in the isotope dataset. In $\mathrm{H}$, larger ELM particle losses due to higher ELM frequency at same input power and gas rate could possibly contribute to the observed lower pedestal density. The interpretative EDGE2D-EIRENE simulations, with simultaneous upstream and outer divertor target profile constraints, indicate higher separatrix electron temperature in $\mathrm{H}$ than in $\mathrm{D}$ for a pair of discharges at similar stored energy (which required higher input power in $\mathrm{H}$ than in $\mathrm{D}$ at the same gas rate). The isotope dependence of linear MHD pedestal stability is small, but if a higher separatrix temperature is considered in $\mathrm{H}$ than in $\mathrm{D}$, this leads to destabilisation of Peeling-Ballooning modes and shrinking of the stability boundary, qualitatively consistent with the reduced pedestal confinement in $\mathrm{H}$.

\section{Introduction}

Unravelling the isotope dependence of plasma confinement and transport would improve our ability to predict the performance of JET and ITER plasmas with deuterium-tritium (DT) mixture. The foreseen plasma scenario for these experiments is H-mode [3], where the level of energy and particle transport at the plasma edge is reduced and a steep pressure gradient is formed, which gives rise to a pressure pedestal. Although positive isotope mass scaling of the thermal energy confinement time in H-mode plasmas has been observed in several tokamaks such as JET [1], JT-60U [4, 5, 6], DIII-D [7] and ASDEX-Upgrade $[8,9,10]$, this favourable isotope dependence has not yet been fully understood theoretically.

H-mode experiments in JET with the carbon wall (JET-C) with different hydrogen isotopes showed virtually no isotope dependence of the thermal energy confinement time in plasmas at similar density: $\tau_{\mathrm{E}, \mathrm{th}} \propto A^{0.03}$, where $A$ the mass number of the main ion $\left(A=m_{\text {ion }} / m_{\text {proton }}\right)[11]$. The strong positive isotope dependence of the pedestal stored energy was compensated with a weak negative isotope dependence of the core plasma [11]. A possible explanation for the low exponent is due to the collinearity of the density and mass dependence in the dataset. This is confirmed by a recent analysis of Maslov [12], which analysed the JET-C dataset in detail. The regression analysis in [12] reports $\tau_{\mathrm{E}, \mathrm{th}} \propto A^{0.2}$ for a JET-C dataset of $\sim 1000$ samples, which includes Hydrogen $(\mathrm{H})$, Deuterium (D), Tritium (T) and DT plasmas at all densities.

JT-60U found that a strong positive isotope dependence of the thermal energy confinement time arises from the core and the pedestal structure has no dependence on the isotope mass $[4,5,6]$. Studies on ASDEX-Upgrade have also reported that roughly a factor of 2 higher heating power is required to match the pedestal pressure in $\mathrm{H}$ and $\mathrm{D}[10]$.

Recent isotope experiments in $\mathrm{H}$ and D plasmas in JET with the ITER-like Wall (JET-ILW) showed a doubling of the power threshold for type III/type I ELMs from D to $\mathrm{H}$ [1]. In type I ELMy H-mode plasmas a positive scaling of $\tau_{\mathrm{E}, \mathrm{th}}$ with the isotope mass was observed: $\tau_{\mathrm{E}, \mathrm{th}} \propto A^{0.4}[1,13]$. The gradient length $\left(R / L_{T}\right.$, where $R$ is the 
major radius, $L_{T}=T / \nabla T$ and $T$ is the temperature) of core temperature profiles was similar in $\mathrm{H}$ and $\mathrm{D}$, despite large variations in the heat flux $[1,13]$. This suggests that the isotope effect may originate in the pedestal in conditions where ions and electrons are collisionally coupled and electron and/or ion transport are stiff [1]. At the same input power and fuelling gas rate, the pedestal pressure is typically reduced in $\mathrm{H}$ compared to $\mathrm{D}$, primarily due to lower pedestal density in $\mathrm{H}$. Note that, similar gas fuelling rate in $\mathrm{H}$ and $\mathrm{D}$ does not necessarily lead to similar main chamber neutral pressure with the two isotopes, but due to lack of measurements any difference could not be quantified. In $\mathrm{H}$ and D plasmas at the same pedestal pressure, which requires roughly double input power in $\mathrm{H}$ than in $\mathrm{D}$ at same gas injection, the low pedestal density in $\mathrm{H}$ is compensated by higher temperature. These observations suggest that understanding the reduced particle transport with increasing isotope mass is crucial to explain the isotope effect in the JETILW pedestal.

This paper examines the isotopic dependence of the type I ELMy H-mode pedestal. In particular, the pedestal structure, edge transport and the isotope effect on linear MHD stability are analysed in a series of JET-ILW H and D type I ELMy H-mode plasmas. The pedestal structure analysis found that at the same input power the pedestal electron pressure gradient is typically lower in $\mathrm{H}$ than in $\mathrm{D}$ at similar pedestal pressure width. The pedestal density width is narrower (or similar) in $\mathrm{H}$ than in $\mathrm{D}$, which contradicts the neutral penetration model [2] and suggests that transport may also play a role in setting the shape of the density pedestal. ELM energy and particle losses are compared in $\mathrm{H}$ and D H-modes using Thomson scattering measurements [14], the stored energy signal from EFIT magnetic equilibrium reconstructions, and for particle losses, interferometry [15]. The ELM losses are dominated by particle losses both in $\mathrm{H}$ and $\mathrm{D}$ and the larger ELM frequency in $\mathrm{H}$ than in $\mathrm{D}$ at similar net power may contribute to the lower pedestal density observed in $\mathrm{H}$. The analysis is supported with a set of interpretative EDGE2D-EIRENE simulations [16, 17, 18] for the pedestal and scrape-of-layer (SOL), which shows that higher anomalous perpendicular transport coefficients are needed to explain the reduced pedestal confinement in H. EDGE2D-EIRENE simulations indicate higher separatrix temperature in $\mathrm{H}$ than in $\mathrm{D}$ for a pair of discharges at similar stored energy (requiring higher input power in $\mathrm{H}$ than in $\mathrm{D}$ at low gas rate). Linear MHD stability is examined with the HELENA fixed boundary equilibrium [19] and ELITE ideal MHD stability [20,21] codes and diamagnetic stabilisation is taken into account, which is shown to introduce a small, but favourable isotope effect. As the separatrix temperature is used to radially align the kinetic profiles to the last closed flux surface (LCFS), a higher separatrix temperature in $\mathrm{H}$ results in an outward radial shift of the peak pressure gradient with respect to the $\mathrm{D}$ counterpart, which destabilises peelingballooning (P-B) modes $[20,21]$ leading to a degraded pedestal in $\mathrm{H}$, consistent with experimental observations.

The paper is organised as follows. Section 2 describes the data set of JET-ILW H and D type I ELMy H-modes used in this work with the characterisation of the pedestal structure and discussing neutral penetration for the edge density profile. ELM energy 
and particle losses are analysed in section 3. Investigation of the edge plasma and the scrape-off layer using interpretative EDGE2D-EIRENE simulations is presented in section 4 . In section 5, the isotope effect on pedestal stability is examined. A summary and conclusions are presented in section 6 .

\section{Pedestal structure in JET-ILW $H$ and D type I ELMy H-modes}

In the present paper JET-ILW type I ELMy H-modes at low plasma triangularity $(\delta \approx 0.2)$ with mostly NBI heating are analysed. The clear separation between type I and type III ELMy H-modes in these plasmas has already been discussed in [1]. The dataset includes power $\left(P_{\mathrm{NBI}}=3-16 \mathrm{MW}\right)$ and gas scans at two different plasma current and magnetic field combinations $\left(1.0 \mathrm{MA} / 1.0 \mathrm{~T}\right.$ with $q_{95}=3.0$ and $1.4 \mathrm{MA} / 1.7 \mathrm{~T}$ with $\left.q_{95}=3.7\right)$. Hereinafter, the $H_{2} / D_{2}$ gas rates will be referred to as "low" $=3-4.5 \cdot 10^{21} \mathrm{e} / \mathrm{s}$, "medium" $=8-10 \cdot 10^{21} \mathrm{e} / \mathrm{s}$ and "high" $=16-18 \cdot 10^{21} \mathrm{e} / \mathrm{s}$. NBI was operated with the ion species of the plasma (H-NBI in $\mathrm{H}$ and D-NBI in D plasmas). The plasma purity was higher than $97 \%$ both in $\mathrm{H}$ and $\mathrm{D}$.

The majority of the dataset is in the so-called "Corner" or C/C divertor configuration, where both divertor strike points are close to the pumping duct. One power scan (1.4 MA/1.7 T, at low gas rate) is in the so-called $\mathrm{V} / \mathrm{H}$ configuration, where the inner strike point is on the vertical target and outer strike point is on the horizontal target. Due to a 2-3 fold increase in sub-divertor neutral pressure, and thus improved cryo-pumping, operation in $\mathrm{C} / \mathrm{C}$ configuration leads to a $10-15 \%$ decrease in $n_{\mathrm{e}, \mathrm{PED}}$ and a similar increase in $T_{\mathrm{e}, \mathrm{PED}}$ at similar $p_{\mathrm{e}, \mathrm{PED}}$ values than in $\mathrm{V} / \mathrm{H}$ configuration [22]. In the present section, the two different divertor configurations are treated together as the main scope of this paper is to present the differences between $\mathrm{H}$ and $\mathrm{D}$ plasmas. The effect of divertor configuration on the pedestal parameters has already been published in $[22]$.

The pedestal structure in the D plasmas of the 1.4 MA/1.7 T dataset has already been characterised elsewhere [22, 23], but here some of those findings are recalled for comparison with the $\mathrm{H}$ plasmas. First, the ELM frequency $\left(f_{\mathrm{ELM}}\right)$ as a function of the power crossing the separatrix $\left(P_{\text {sep }}\right)$ is shown in figure 1 to demonstrate the type I nature of the ELMs and to show some other important features in the dataset. $P_{\text {sep }}$ is defined as follows,

$$
P_{\text {sep }}=P_{\text {loss }}-P_{\text {rad,bulk }}=P_{\text {abs }}-\mathrm{d} W / \mathrm{d} t-P_{\text {rad,bulk }},
$$

where $P_{\text {abs }}$ is the total absorbed power given by the sum of the Ohmic power, the absorbed neutral beam power (accounting for shine through) and absorbed ion cyclotron

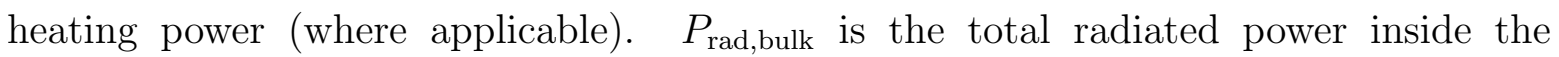
separatrix as estimated by a weighted sum of representative bolometer channels, $P_{\text {loss }}$ is the loss power given by $P_{\text {loss }}=P_{\text {abs }}-\mathrm{d} W / \mathrm{d} t$ and $\mathrm{d} W / \mathrm{d} t$ is the rate of change of the total stored energy, which is negligible in the steady phases of the discharges.

In the $1.4 \mathrm{MA} / 1.7 \mathrm{~T}$ dataset $f_{\mathrm{ELM}}$ increases with $P_{\text {sep }}$ and $f_{\mathrm{ELM}}$ is higher in $\mathrm{H}$ than in $\mathrm{D}$ at a low gas rate at given input power (figure 1a). Note that NBI heating was 
limited to $10 \mathrm{MW}$ in $\mathrm{H}$, thus in the "medium" and "high" gas H plasmas at 1.4 MA/1.7 T, 2-5 MW ion cyclotron resonance heating (ICRH: $51 \mathrm{MHz}, \mathrm{H}$ majority, 2nd harmonic) was added to the heating mix to reach type I ELMy H-modes, which is expected to lead to an increase in $f_{\text {ELM }}$ compared to NBI only plasmas [24]. All other plasmas were NBI heated only.

At 1.0 MA/1.0 T two different "low" gas levels are distinguished: "very low" = $3 \cdot 10^{21} \mathrm{e} / \mathrm{s}$ and "low" $=4.5 \cdot 10^{21} \mathrm{e} / \mathrm{s}$. As it is visible in figure $1 \mathrm{~b}, f_{\mathrm{ELM}}$ decreases with $P_{\text {sep }}$ for the "low gas" power scan and is constant for the "high gas" power scan. Despite the $f_{\text {ELM }}$ behaviour, other parameters such as $\beta_{N}$ and the ELM signature in the divertor Be II photon flux suggest that these pulses are in the type I ELMy regime. It is possible that the decreasing trend of $f_{\text {ELM }}$ with the input power for the "low gas" dataset is a consequence of the density being very close to the point where the type IIItype I ELMy power threshold ( $P_{\mathrm{III}-\mathrm{I}}$ ) "rolls over" from the low density branch to the high density branch and the small density variation between pulses could lead to some plasmas accessing type I ELMy H-mode from the low density branch, while others from the high density branch. In the low density branch, $P_{\text {III-I }}$ increases very rapidly with decreasing density. Thus, a given $P_{\text {sep }}$ is closer to the type III to type I power threshold in the low than in the high density branch. This may lead to lower $f_{\mathrm{ELM}}$ in the low density branch than in the high density branch even at higher $P_{\text {sep }}$, resulting in the observed decreasing trend of $f_{\mathrm{ELM}}$ with the input power. In $\mathrm{D}$, at $1.0 \mathrm{MA} / 1.0 \mathrm{~T}$ stable $\mathrm{H}$-mode operation was not possible at "low gas" rate due to $\mathrm{W}$ and mid-Z impurity accumulation in the core. The "medium gas" power scan with a factor of 2 variation in $P_{\text {sep }}$ provides a good basis for comparison between $\mathrm{H}$ and $\mathrm{D}$ at this plasma current $\left(I_{p}\right)$ and magnetic field $\left(B_{t}\right)$, thus the analysis of the 1.0 MA/1.0 T dataset will mostly focus on these data.
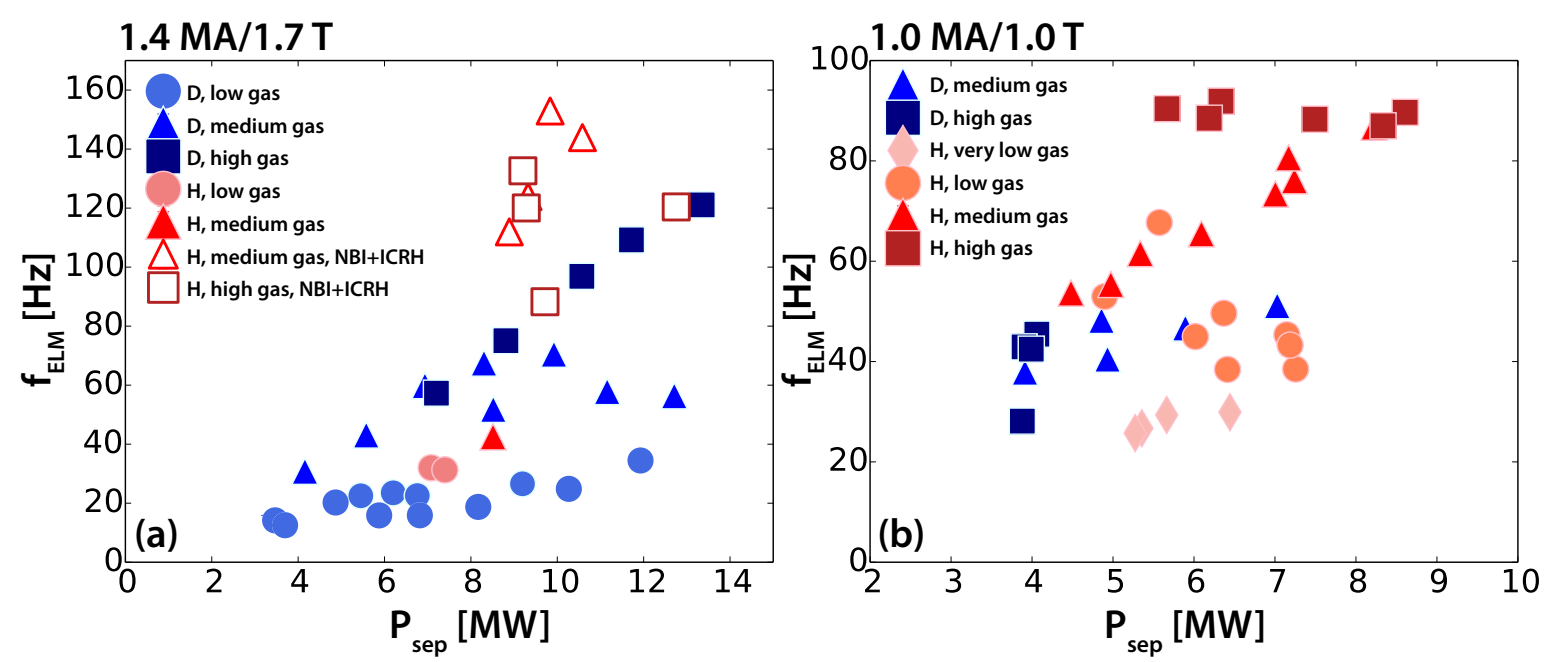

Figure 1. ELM frequency as a function of net power crossing the separatrix for the (a) $1.4 \mathrm{MA} / 1.7 \mathrm{~T}$ and the (b) 1.0 MA/1.0 T dataset. Note that medium and high gas pulses at $1.4 \mathrm{MA} / 1.7 \mathrm{~T}$ (open symbols on figure (a)) above $P_{\text {sep }}=8.5 \mathrm{MW}$ were heated also with ICRH. 
The pedestal structure analysis is carried out for the pre-ELM phase (namely the last $20 \%$ of the ELM cycle) and is based on the mtanh [25] fitted electron density $\left(n_{e}\right)$ and temperature $\left(T_{e}\right)$ profiles as measured by Thomson scattering (TS). The kinetic profiles collected from a steady time window $\left(>10 \times \tau_{\mathrm{E}, \mathrm{th}}\right)$ of the discharge are ELMsynchronised to improve signal statistics $[26,27]$. The width and height of the pedestal electron density and temperature are taken directly from the mtanh fit. The error bars on the pedestal structure parameters are defined as the standard deviation of the parameter estimates of the mtanh fit.

\subsection{Pedestal height}

Figure 2 shows the electron pedestal pressure $\left(p_{e, P E D}[\mathrm{~Pa}]=1.602 \times n_{e, P E D}\left[10^{-19} \mathrm{~m}^{-3}\right] \times\right.$ $T_{e, P E D}[\mathrm{eV}]$, where 1.602 originates from the elementary charge as $T_{e}$ is measured in $\mathrm{eV}$ ) as a function of $P_{\text {sep }}$. Both at 1.0 MA/1.0 T and 1.4 MA/1.7 T, $p_{\mathrm{e}, \mathrm{PED}}$ decreases with increasing gas rate both in $\mathrm{H}$ and $\mathrm{D}$. In general, $p_{\mathrm{e}, \mathrm{PED}}$ is higher in $\mathrm{D}$ than in $\mathrm{H}$ at a given $P_{\text {sep }}$.
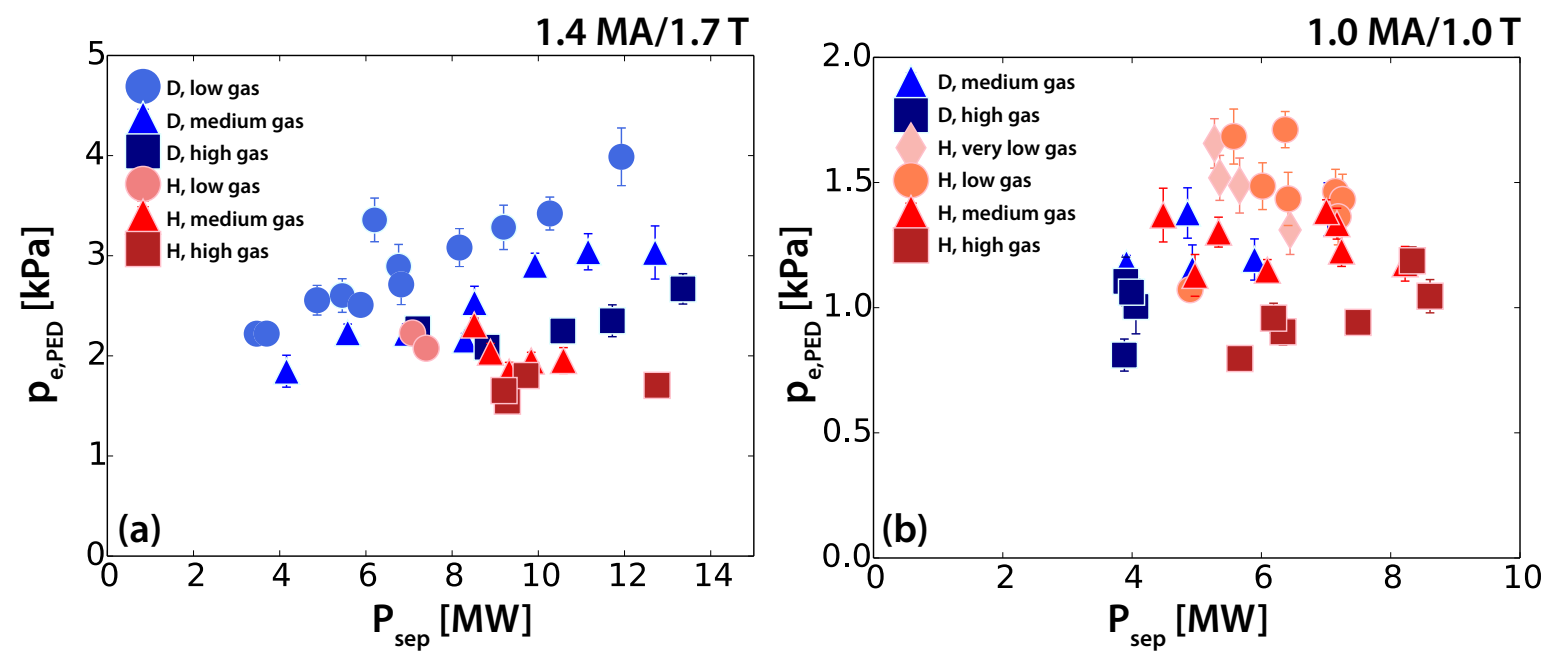

Figure 2. The electron pressure at the pedestal top as a function of net power crossing the separatrix for the (a) $1.4 \mathrm{MA} / 1.7 \mathrm{~T}$ and the (b) $1.0 \mathrm{MA} / 1.0 \mathrm{~T}$ dataset.

At 1.0 MA/1.0 T and medium gas rate $p_{\mathrm{e}, \mathrm{PED}}$ is comparable in $\mathrm{H}$ than in $\mathrm{D}$, but the total thermal stored energy is still higher in $\mathrm{D}$. This is due to higher $T_{e}$ peaking in $\mathrm{D}$ than in $\mathrm{H}$, when the core temperature is compared to $T_{\mathrm{e}, \mathrm{PED}}$ as shown in figure $3 \mathrm{a}$. However, when $T_{e}$ peaking is defined as $T_{e}\left(\rho_{\mathrm{TOR}}=0.3\right) / T_{e}\left(\rho_{\mathrm{TOR}}=0.8\right)$ (with $\rho_{\mathrm{TOR}}$ the normalised toroidal flux), the difference between $\mathrm{H}$ and $\mathrm{D}$ diminishes as shown in figure $3 \mathrm{~b}$, which is consistent with $R / L_{T_{e}}$ being similar at $\rho_{\text {TOR }}=0.5$ as shown in figure 18 in [1]. This can also be seen in figure 4 , where the electron kinetic profiles on a log scale are shown for a pair of $\mathrm{H}$ and D pulses at the same $P_{\text {sep }}$ as a representative example. Gradient lengths in the core are very similar and any difference in the temperature gradient length arises at $\rho_{\mathrm{TOR}}>0.8$. Therefore, for the $1.0 \mathrm{MA} / 1.0 \mathrm{~T}$ medium gas rate dataset as well, the difference in thermal energy confinement between $\mathrm{H}$ and $\mathrm{D}$ is emerging at the edge, but 
in this case it is not well represented by the pedestal top values as derived from the mtanh fit. This may be due to the lack of TS data in $\mathrm{H}$ just inside the pedestal, which could lead to higher uncertainties in the profile fitting method.
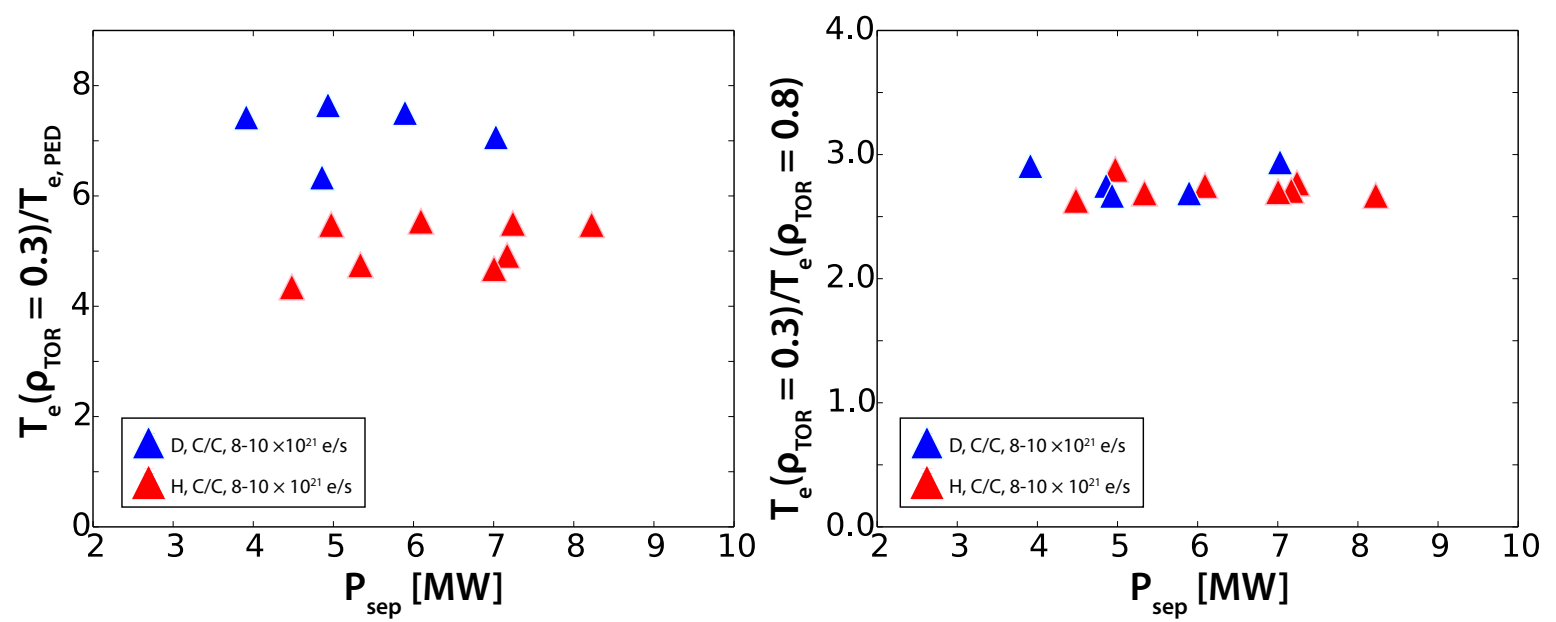

Figure 3. $T_{e}$ peaking in the 1.0 MA/1.0 T medium gas dataset defined in two different ways: (a) $T_{e}\left(\rho_{\mathrm{TOR}}=0.3\right) / T_{\mathrm{e}, \mathrm{PED}}$ and $(\mathrm{b}) T_{e}\left(\rho_{\mathrm{TOR}}=0.3\right) / T_{e}\left(\rho_{\mathrm{TOR}}=0.8\right)$.
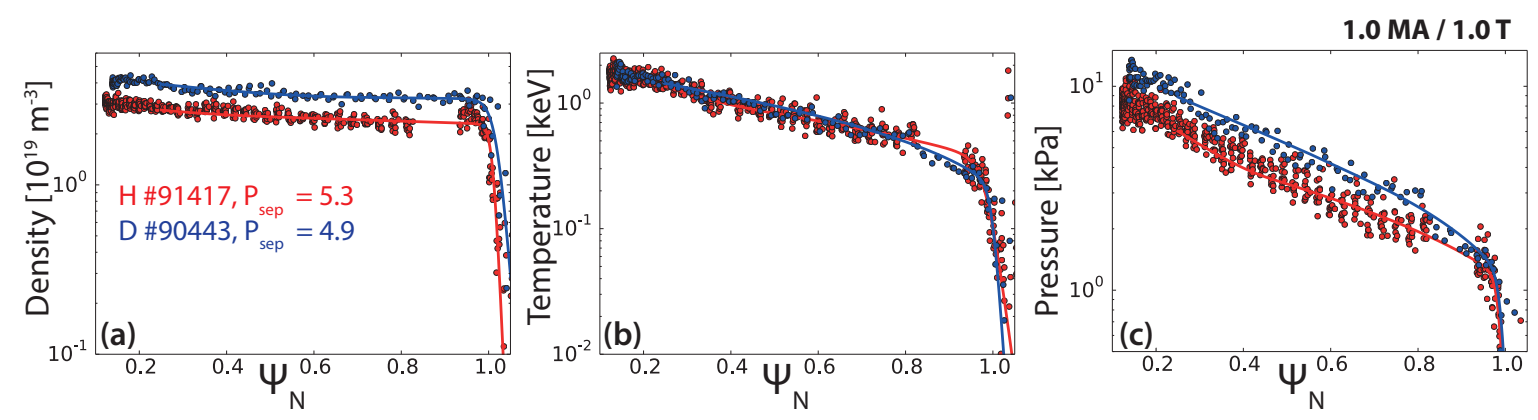

Figure 4. pre-ELM (80-100\% of the ELM cycle) electron kinetic profiles from TS for a pair of $\mathrm{H}$ (\#91417, in red) and D (\#90443, in blue) pulses at 1.0 MA/1.0 $\mathrm{T}$ at the same $P_{\text {sep }}$ as a representative example. (a) Electron density, (b) electron temperature and (c) electron pressure are shown on a log scale to compare the gradient lengths in the core. Profiles are radially shifted to have $T_{\mathrm{e}, \mathrm{sep}}=100 \mathrm{eV}$.

The ion temperature $\left(T_{i}\right)$ is similar to $T_{e}$ at the pedestal top within the measurement uncertainties of the edge Charge Exchange Recombination Spectroscopy (CXRS) system. Discharges with good $T_{i}$ data in the pedestal gradient region confirm $T_{e} \approx T_{i}$, although $T_{i}$ at the separatrix cannot be resolved. The line-averaged $Z_{\text {eff }}$ in the 1.4 MA/1.7 $\mathrm{T}$ dataset varies between 1.1 and 1.5 for $\mathrm{D}$ and between 1.2 and 1.8 for H. For the 1.0 MA/1.0 T dataset it varies between 1.2 and 1.4 for $\mathrm{D}$ and between 1.1 and 1.4 for $\mathrm{H}$. Assuming Be as single impurity and $T_{e}=T_{i}$, the ion dilution leads to at most $10 \%$ difference between the total pressure (calculated as $p=p_{e}+p_{i}$ ) and $2 \times p_{e}$, thus the conclusions drawn from the analysis of the electron pressure also apply to the total pressure. The edge $n_{e}-T_{e}$ diagram in figure 5 shows that the pedestal density is 
typically lower in $\mathrm{H}$ than in D. Pedestals at the same $p_{\mathrm{e}, \mathrm{PED}}$ (typically obtained with more heating power in $\mathrm{H}$ than in $\mathrm{D}$ ) have lower density, but higher temperature in $\mathrm{H}$ compared to D. In other words, by varying input power and/or gas rate it was not possible to simultaneously match $n_{e}$ and $T_{e}$ in $\mathrm{H}$ and $\mathrm{D}$, as for example in JT-60U experiments $[4,5,6]$.

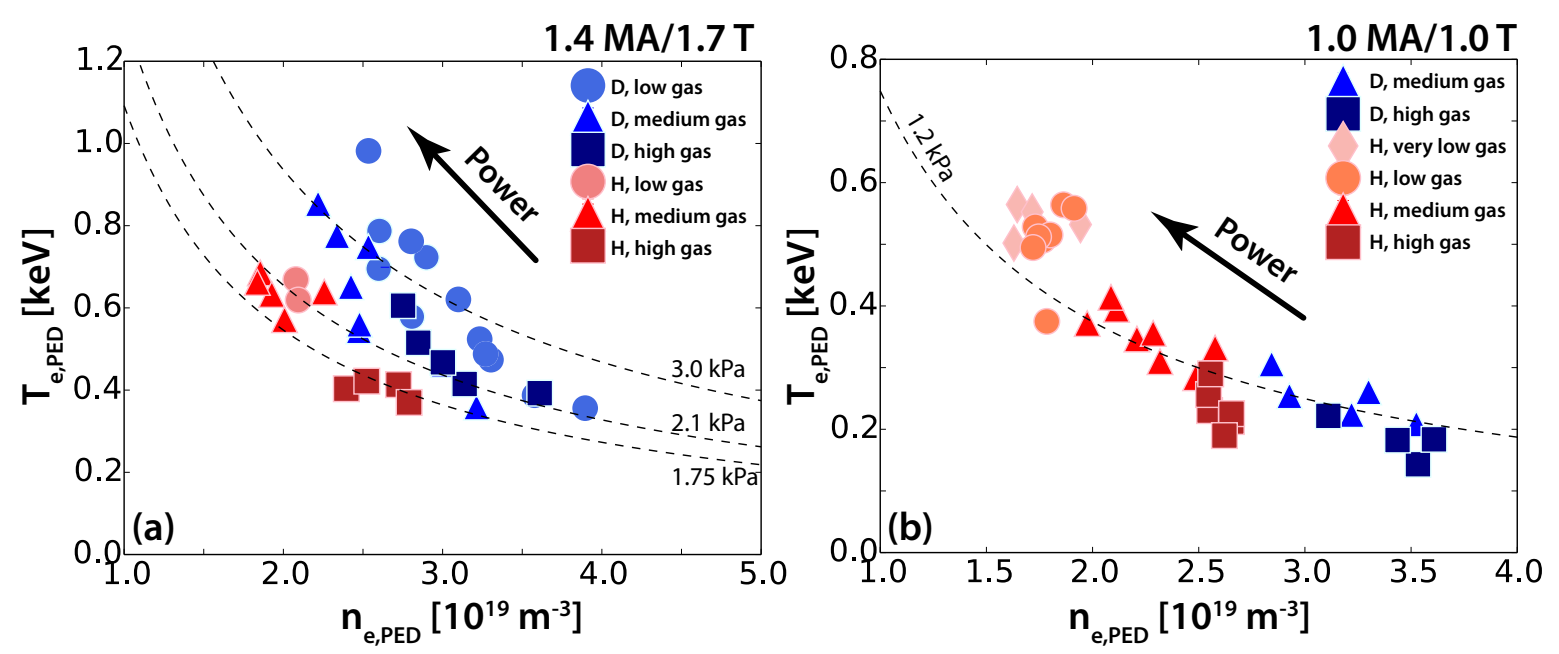

Figure 5. $n_{\mathrm{e}, \mathrm{PED}}-T_{\mathrm{e}, \mathrm{PED}}$ diagram for the (a) $1.4 \mathrm{MA} / 1.7 \mathrm{~T}$ and the (b) $1.0 \mathrm{MA} / 1.0 \mathrm{~T}$ dataset. The dashed black lines are isobars at a pressure level indicated in the figure.

\subsection{Pedestal gradient}

The peak pedestal pressure gradient is comparable in $\mathrm{H}$ and $\mathrm{D}$ within error bars at the same pedestal top poloidal beta $\left(\beta_{\text {pol,PED }}\right)$ at both plasma current levels, as shown in figure 6. However, at 1.4 MA/1.7 $\mathrm{T} \nabla n_{e}$ is lower and $\nabla T_{e}$ is higher in $\mathrm{H}$ compared to D (see figure $7 \mathrm{a}$ for $\nabla n_{e}$ and figure $8 \mathrm{a}$ for $\nabla T_{e}$ ), while at $1.0 \mathrm{MA} / 1.0 \mathrm{~T} \nabla n_{e}$ and $\nabla T_{e}$ are comparable in $\mathrm{H}$ and $\mathrm{D}$ (see figure $7 \mathrm{~b}$ for $\nabla n_{e}$ and figure $8 \mathrm{~b}$ for $\nabla T_{e}$ ). At $1.4 \mathrm{MA} / 1.7 \mathrm{~T}$, $\nabla p_{e}$ increases with increasing $\beta_{\mathrm{pol}, \mathrm{PED}}$ and more power is needed in $\mathrm{H}$ than in $\mathrm{D}$ to reach the same $\beta_{\text {pol,PED }}$, thus $\nabla p_{e}$ is typically larger in $\mathrm{D}$ than in $\mathrm{H}$ at the same $P_{\text {sep }}$.

\subsection{Pedestal width}

The pedestal pressure width, defined here as $\Delta n_{e} / 2+\Delta T_{e} / 2 \ddagger$, has a much larger variation at a given $\beta_{\text {ped,pol }}$ than is expected by the EPED model $\left(\Delta p_{\mathrm{e}, \mathrm{PED}}=c_{\mathrm{EPED}} \times \beta_{\mathrm{pol}, \mathrm{PED}}^{1 / 2}\right.$ with $\left.c_{\mathrm{EPED}}=0.076[28]\right)$ both in $\mathrm{H}$ and $\mathrm{D}$ as it is shown in figure $9 . \beta_{\mathrm{pol}, \mathrm{PED}}$ is the poloidal normalised pressure at the pedestal top calculated using the expression given by $[29,30]$. At 1.4 MA/1.7 T $\Delta p_{e}$ broadens with increasing gas rate at constant $\beta_{\text {ped,pol, which is }}$ not consistent with EPED (with constant width multiplier $c_{\text {EPED }}$ ) [30, 23]. No clear trend with $\beta_{\mathrm{pol}, \mathrm{PED}}$ is observed in the variation of $\Delta p_{e}$ at $1.0 \mathrm{MA} / 1.0 \mathrm{~T}$. The difference

$\ddagger$ The EPED pedestal pressure width definition [28] is used here for comparison with the EPED pedestal width scaling. $\Delta n_{e}$ and $\Delta T_{e}$ are the full widths of the mtanh profiles, not only the part up to the LCFS. 

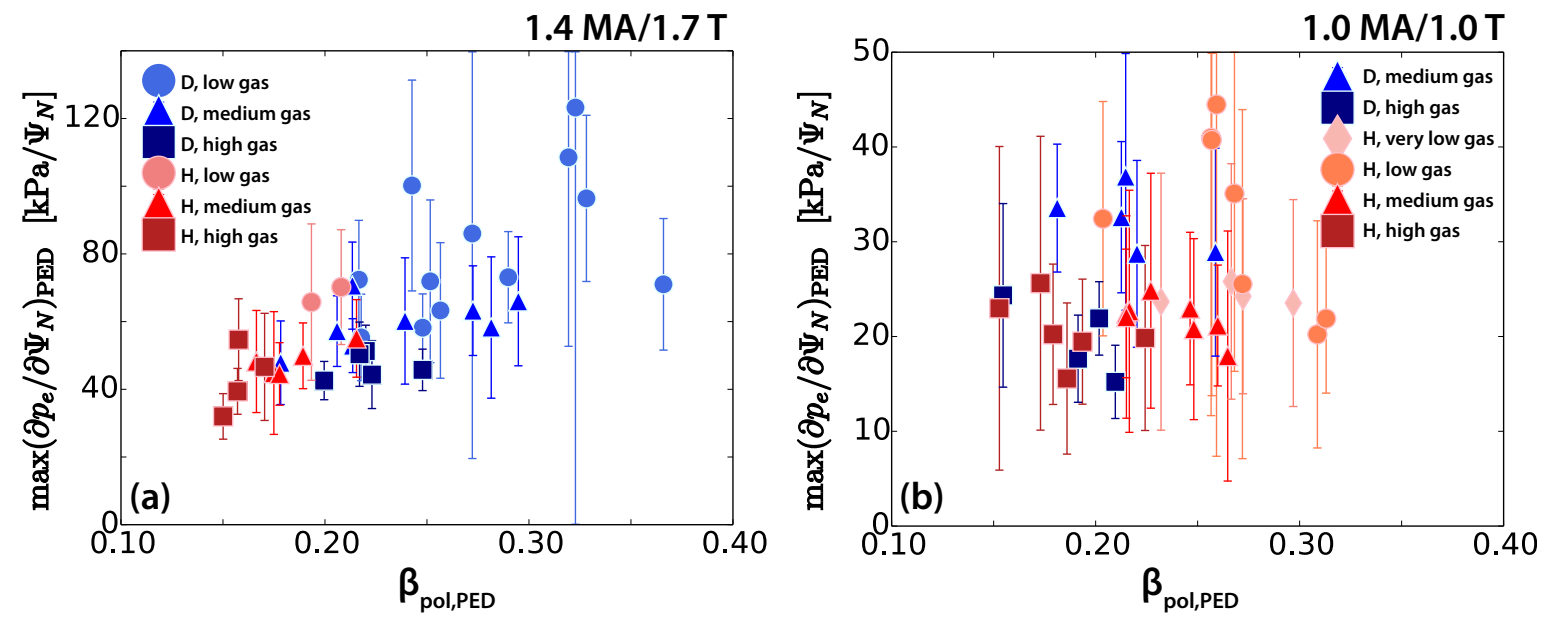

Figure 6. The peak electron pressure pedestal gradient $\left(\nabla p_{e}\right)$ as a function of $\beta_{\mathrm{pol}, \mathrm{PED}}$ for the (a) $1.4 \mathrm{MA} / 1.7 \mathrm{~T}$ and the (b) $1.0 \mathrm{MA} / 1.0 \mathrm{~T}$ dataset.
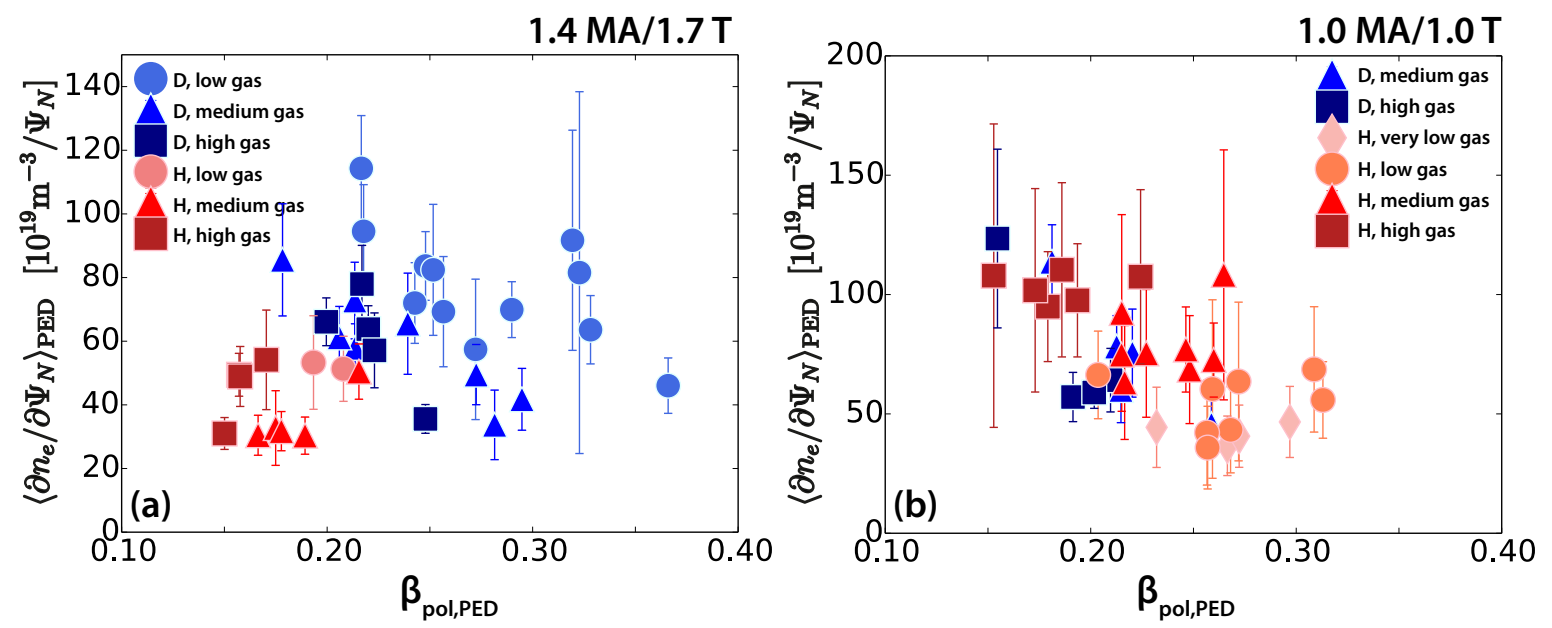

Figure 7. The average density pedestal gradient $\left(\nabla n_{e}\right)$ as a function of $\beta_{\text {pol,PED }}$ for the (a) $1.4 \mathrm{MA} / 1.7 \mathrm{~T}$ and the (b) $1.0 \mathrm{MA} / 1.0 \mathrm{~T}$ dataset. $\nabla n_{e}=n_{\mathrm{e}, \mathrm{PED}} / \Delta n_{e}$.

in $p_{\mathrm{e}, \mathrm{PED}}$ between $\mathrm{H}$ and $\mathrm{D}$ at the same $P_{\text {sep }}$ at $1.4 \mathrm{MA} / 1.7 \mathrm{~T}$ (shown in figure $2 \mathrm{a}$ ) is mainly due to lower $\nabla p_{e}$ in $\mathrm{H}$ than in $\mathrm{D}$ and similar pedestal $p_{e}$ width.

\subsection{Edge density profile and neutral fuelling}

It is anticipated that the penetration of neutrals and the resulting particle source at the edge of the plasma could be an important mechanism in setting the density pedestal. Indeed, the neutral penetration model (NPM) assumes that the pedestal density is set by the edge particle flux and that the pedestal width is approximately equal to the neutral penetration length [2]. This model is tested here against the experimental pedestal density width of $\mathrm{H}$ and $\mathrm{D}$ plasmas. At similar temperature, $\mathrm{H}$ neutrals have a higher thermal speed than D neutrals, thus larger mean free path and neutral penetration 

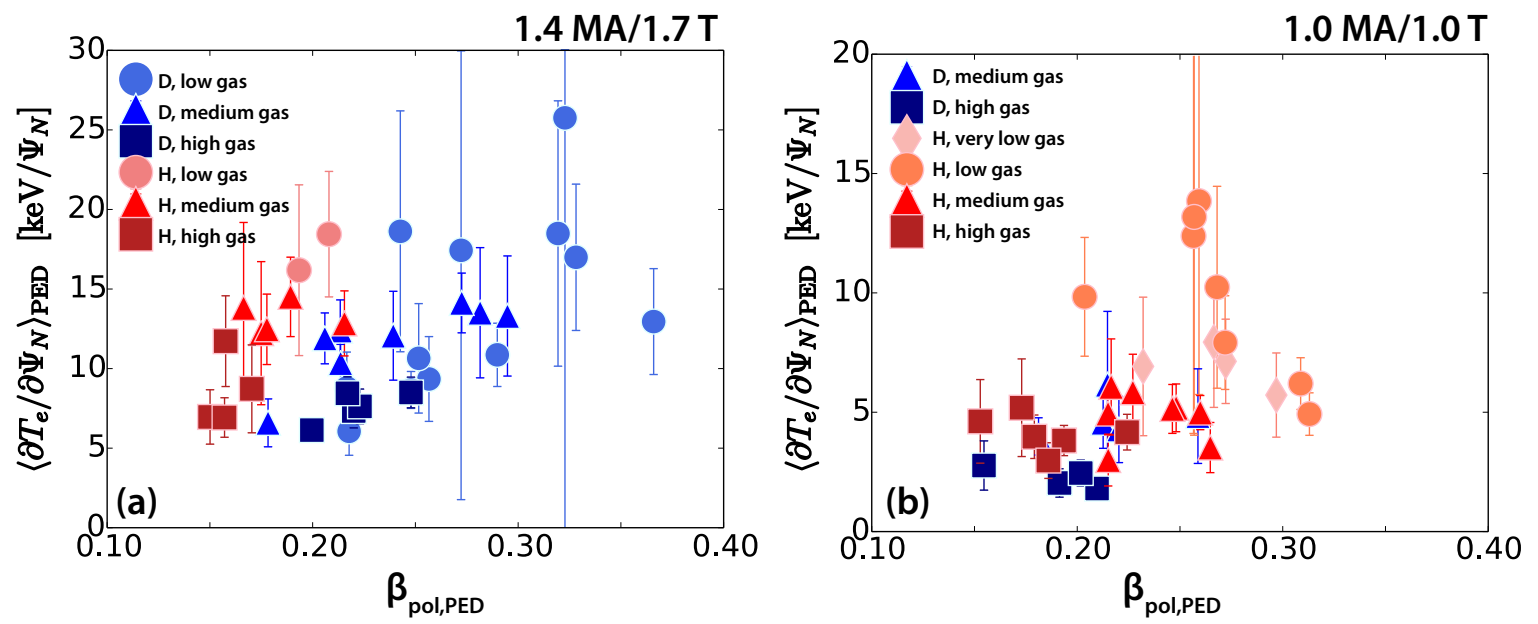

Figure 8. The average electron temperature pedestal gradient $\left(\nabla T_{e}\right)$ as a function of $\beta_{\text {pol,PED }}$ for the (a) $1.4 \mathrm{MA} / 1.7 \mathrm{~T}$ and the (b) $1.0 \mathrm{MA} / 1.0 \mathrm{~T}$ dataset. $\nabla T_{e}=$ $T_{\mathrm{e}, \mathrm{PED}} / \Delta T_{e}$.
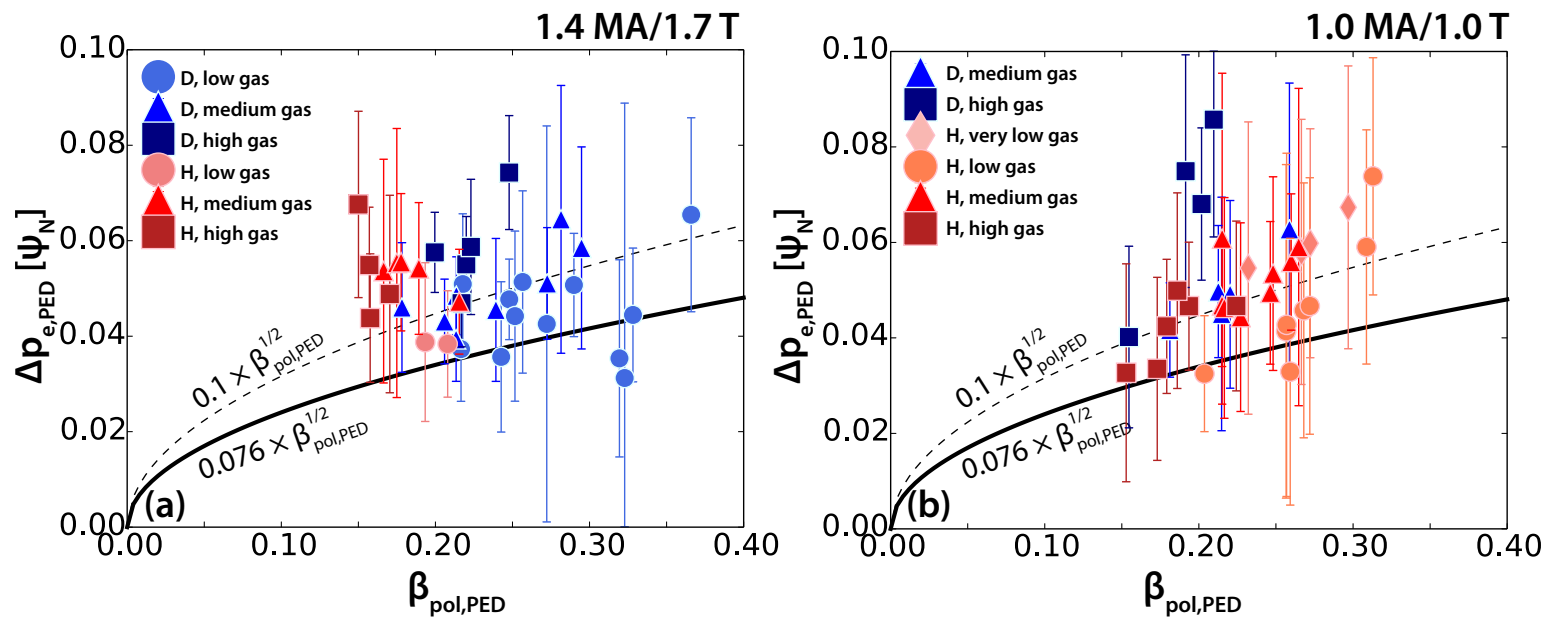

Figure 9. The electron pressure pedestal width in normalised poloidal flux $\left(\Psi_{N}\right)$ for the (a) $1.4 \mathrm{MA} / 1.7 \mathrm{~T}$ and the (b) $1.0 \mathrm{MA} / 1.0 \mathrm{~T}$ dataset. The solid black lines indicate the $0.076 \times \beta_{\text {pol,PED }}^{1 / 2}$ EPED scaling [28] and the dashed black lines indicate the $0.1 \times \beta_{\text {pol,PED }}^{1 / 2}$ curve to show the variation in the dataset.

length is expected in H. According to the NPM this should lead to a wider density pedestal in $\mathrm{H}$.

The neutral penetration model [2] describes the width of the density pedestal as

$$
\Delta_{n_{e}}=\frac{2 V_{N}}{S_{i} E n_{e, p e d}}
$$

where $\Delta_{n_{e}}$ is the pedestal width at the midplane (in real units), $V_{N}$ is the neutral velocity, $E$ is a flux expansion parameter, $n_{e, p e d}$ is the pedestal top density and $S_{i}$ is the ionisation rate, which is approximated as $\sigma_{i} V_{e}[2]$ with $\sigma_{i}$ the cross section for electron impact ionisation and $V_{e}$ the electron thermal velocity. If the fuelling location 
is maintained, $T_{e} / T_{i} \approx$ constant and assuming that the neutrals are in equilibrium with the ions, the NPM predicts the following relation between $\Delta_{n_{e}}$ and $n_{e, p e d}$ :

$$
\Delta_{n_{e}} \sim \frac{1}{\sqrt{A} n_{e, p e d}}
$$

This assumption can easily be tested against the experimental pedestals analysed in the present paper. Previous JET-ILW studies in D plasmas indicated that for some datasets at low $\delta, \Delta_{n_{e}}$ is broadly consistent with the $1 / n_{e, p e d}$ dependence of the pedestal density, while for other cases (for example a high $\delta$ dataset [23] or a dimensionless $\nu^{*}$ scan [31]) the density pedestal broadens at roughly constant $n_{e, p e d}$, in contradiction to the assumptions of the NPM.

In figure $10 \Delta n_{e} \times \sqrt{A}$ is shown as a function of $n_{e, p e d}$ for the $\mathrm{H}$ and $\mathrm{D}$ pedestals discussed in this section. At 1.0 MA/1.0 T (figure 10b), the pedestal width in $\mathrm{H}$ follows the $1 / n_{e, p e d}$ scaling at all fuelling gas rates, but the pedestals are significantly narrower in $\mathrm{H}$ than in D in contradiction to the NPM. Figure 10a shows the comparison for the 1.4 MA/1.7 $\mathrm{T}$ dataset, where the result depends on the gas fuelling rate. At low gas rate (circles), the $\mathrm{H}$ pedestals are narrower than the $\mathrm{D}$ pedestals, in contradiction to the NPM. At medium (triangles) and high (squares) fuelling gas rates, $\Delta n_{e}$ is similar in $\mathrm{H}$ and D in accordance with the NPM. In summary, this comparison of the experimental observations with the NPM suggests that the changes in neutral fuelling due to the change of the isotope mass is not sufficient to fully describe the difference in pedestal density between $\mathrm{H}$ and D. Inter-ELM pedestal transport and/or ELM losses must also play a role, which is also supported by the interpretative EDGE2D-EIRENE simulations discussed in section 4. Results indicate that the difference in the kinetic profiles between $\mathrm{H}$ and $\mathrm{D}$ is due to significantly higher perpendicular particle and heat diffusivities at the edge in $\mathrm{H}$ than in $\mathrm{D}$.
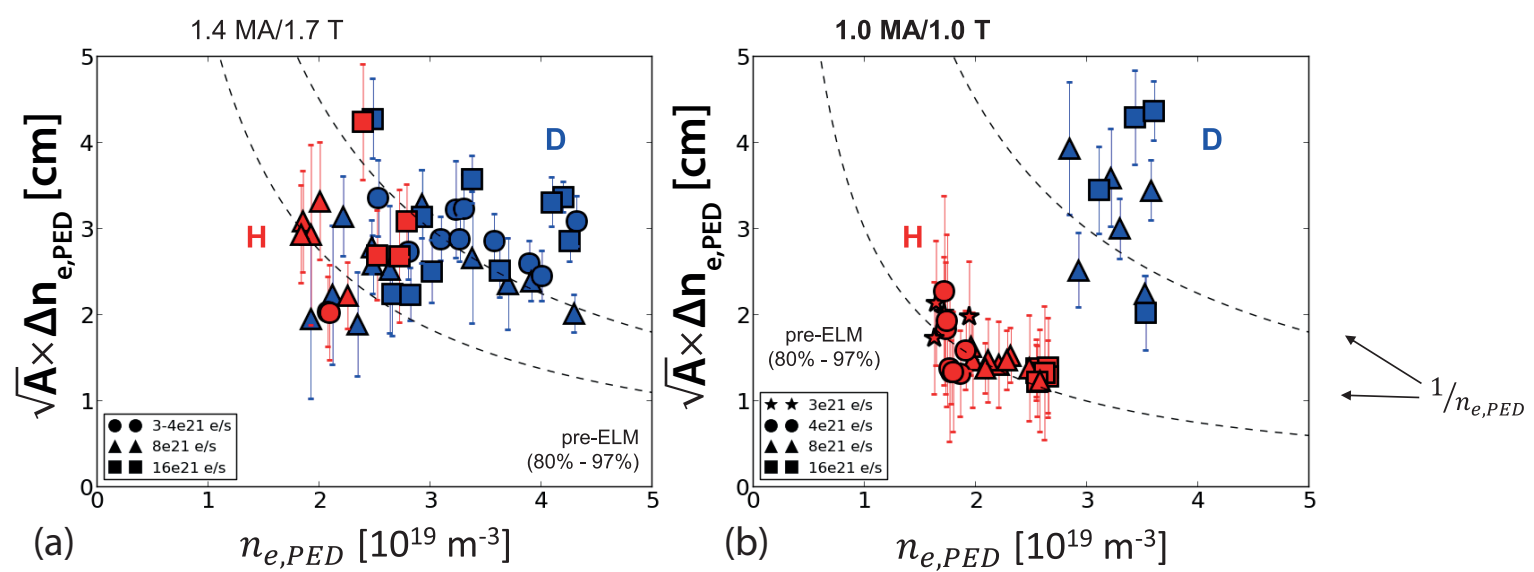

Figure 10. $n_{e, \text { width }} \times \sqrt{A}$ as a function of $n_{e, p e d}$ for the (a) $1.4 \mathrm{MA} / 1.7 \mathrm{~T}$ and the (b) $1.0 \mathrm{MA} / 1.0 \mathrm{~T}$ datasets. 


\subsection{Ratio of the electron density and temperature gradient length, $\eta=L_{n_{e}} / L_{T_{e}}$}

In view of recent gyrokinetic analysis of the pedestal in JET with Carbon wall (JET-C) and JET-ILW $[32,33,34]$, we compare the ratio of the electron density and temperature gradient length $\eta_{e}$ between $\mathrm{H}$ and D plasmas. $\eta=L_{n_{e}} / L_{T_{e}}$, with $L_{n_{e}}=n_{e} / \nabla n_{e}$ and $L_{T_{e}}=T_{e} / \nabla T_{e}$ being the density and temperature gradient lengths, respectively. The growth rate of temperature gradient driven micro turbulence is expected to increase with $\eta_{e}$, generating increasing levels of heat transport inside the pedestal $[32,33]$. It has been reported in [32] and [33] that the degradation of the temperature pedestal in JET-ILW, where significant gas injection is needed for core $W$ control, can partly be explained by an increase in $\eta_{e}$ and $\eta_{i}$ in JET-ILW producing more robust slab-like ion temperature gradient (ITG) and electron temperature gradient (ETG) instability, leading to limited pedestal temperature and demanding more heating power to achieve similar pedestal pressure as in JET-C. The experimental characterisation of $\eta_{e}$ for the JET-ILW $\mathrm{H}$ and D isotope dataset is attempted here, in order to collect any evidence for differences in the inter-ELM transport between $\mathrm{H}$ and $\mathrm{D}$ pedestals, which may shed light on the physics mechanism behind the lower $\mathrm{H}$ pedestals.

$\eta_{e}$ is calculated here from the mtanh fit of the $T_{e}$ and $n_{e}$ TS profiles for the preELM pedestals in a region $0.02 \Psi_{N}$ wide around the centre (peak gradient) of the $T_{e}$ pedestal. $\oint$ Since the variation of $\eta_{e}$ is typically large in the pedestal, in figure 11 ranges of $\eta_{e}$ between the lowest and highest values in the $0.02 \Psi_{N}$ wide region are compared. In the $1.4 \mathrm{MA} / 1.7 \mathrm{~T}$ dataset, there is some separation in $\eta_{e}$ for the discharges at different gas rates in $\mathrm{D}$, as shown in figure 11a. $\eta_{e}$ increases with increasing gas rate for $P_{\text {sep }}>6 \mathrm{MW}$ in $\mathrm{D}$, which is consistent with the larger relative radial shift between $T_{e}$ and $n_{e}$ pedestals at higher gas rates and power $[34,35]$. The position of the $n_{e}$ pedestal is radially outwards with respect to the $T_{e}$ pedestal, thus in the region of maximum $T_{e}$ gradient the density gradient is smaller, leading to higher values of $\eta_{e}$. There is no significant difference between the $\eta_{e}$ values of the $\mathrm{H}$ and $\mathrm{D}$ plasmas (see figure 11a). $L_{n_{e}}$ and $L_{T_{e}}$ at given $P_{\text {sep }}$ are also similar in $\mathrm{H}$ and $\mathrm{D}$ for this dataset. This suggests that the mechanism identified in [32] and [33] explaining the differences between JET$\mathrm{C}$ and JET-ILW pedestals through differences in $\eta_{e}$ does not apply here. Differences in the inter-ELM transport between the $\mathrm{H}$ and $\mathrm{D}$ pedestals due to other mechanisms are not excluded and future work should focus on studying transport with gyrokinetic simulations in order to identify these. Figure $11 \mathrm{~b}$ shows that at $1.0 \mathrm{MA} / 1.0 \mathrm{~T}, \eta_{e}$ is typically larger in $\mathrm{H}$ than in $\mathrm{D}$, which is a result of the $n_{e}$ pedestal being very narrow and shifted radially outwards with respect to the $T_{e}$ pedestal. Higher $\eta_{e}$ in $\mathrm{H}$ may imply differences in pedestal heat transport between $\mathrm{H}$ and $\mathrm{D}$, although the degradation of the pedestal is the least pronounced in this dataset, thus further transport analysis and comparison with gyrokinetic simulations would be required to clarify the relation between the heat transport and $\eta_{e}$ at 1.0 MA/1.0 T.

$\S$ The temperature pedestal width is typically $>0.03 \Psi_{N}$ in the dataset, thus the $0.02 \Psi_{N}$ wide region only covers the gradient region and not the "knee" and the "bottom" of the pedestal. 

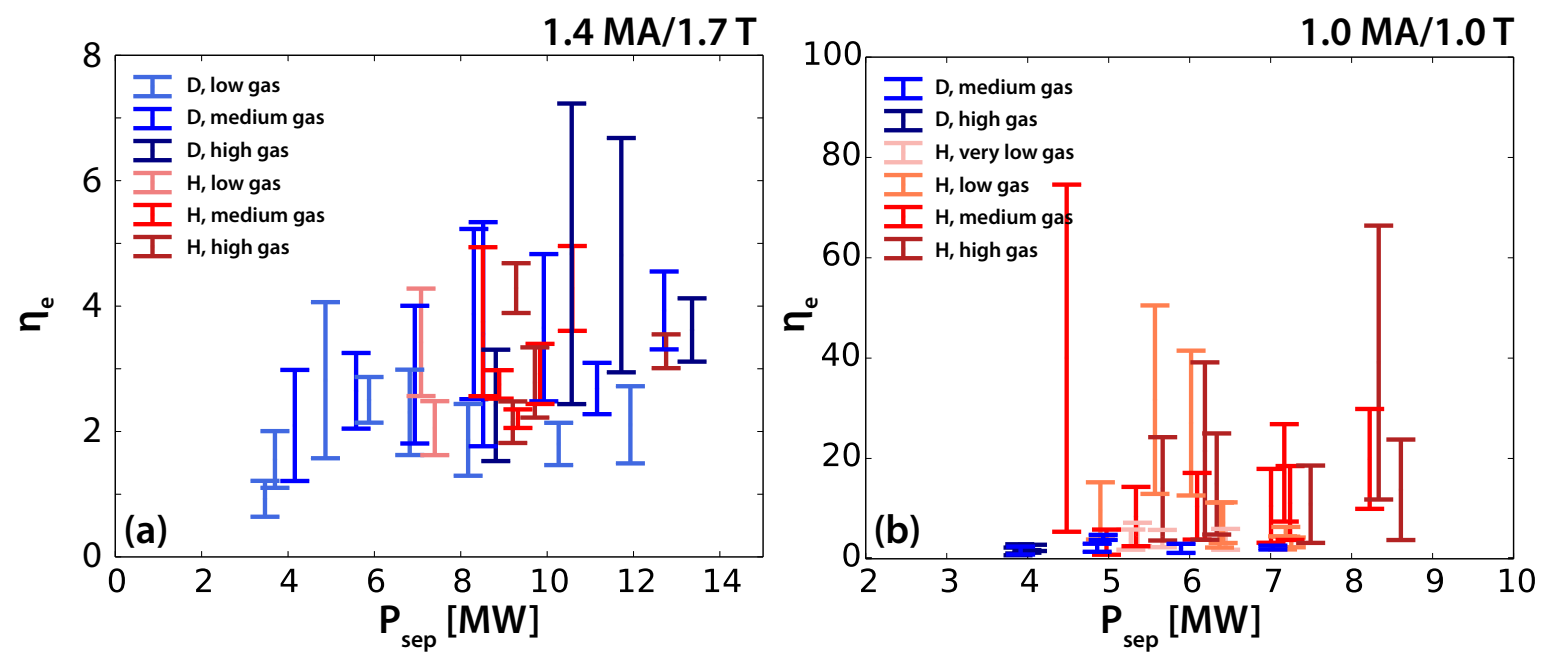

Figure 11. $\eta_{e}=L_{n_{e}} / L_{T_{e}}$ is calculated for the pre-ELM pedestals in a region $0.02 \Psi_{N}$ wide around the centre (peak gradient) of the $T_{e}$ pedestal. The figure shows the range of $\eta_{e}$ between the lowest and highest values in the $0.02 \Psi_{N}$ wide region for the (a) 1.4 MA/1.7 $\mathrm{T}$ and the (b) 1.0 MA/1.0 T datasets.

\section{ELM energy and particle losses}

In this section a power balance analysis is presented to compare $\mathrm{H}$ and $\mathrm{D}$ pulses in terms of radiation, ELM losses and inter-ELM transport. Compared to eq. (1), $P_{\text {sep }}$ in the power balance equation here is separated into inter-ELM and ELM components and $\mathrm{d} W / \mathrm{d} t$ is omitted as only the steady phases of the discharges are examined, where the rate of change of stored energy on time scales longer than the ELM cycle is negligible:

$$
P_{\text {sep }}=P_{\text {inter-ELM }}+P_{\text {ELM }}=P_{\text {loss }}-P_{\text {rad,bulk }}
$$

The ELM energy loss $\left(\Delta W_{\mathrm{ELM}}\right)$ - which gives $P_{\mathrm{ELM}}=\Delta W_{\mathrm{ELM}} \times f_{\mathrm{ELM}}$ - has been evaluated from two independent measurements: a) the stored energy drop from EFIT equilibrium reconstruction $\left(\Delta W_{\mathrm{MHD}}\right)$ and b) Thomson scattering electron kinetic profile measurements $\left(\Delta W_{\mathrm{TS}}\right) . \Delta W_{\mathrm{MHD}}$ is estimated by the difference between the maximum and the minimum of the $W_{\mathrm{MHD}}$ signal in the vicinity of the ELM crash as illustrated by the red arrow in figure 12. $\Delta W_{\mathrm{MHD}}$ is evaluated for all ELMs individually in the steady phase of the discharge. The ELM energy losses are then averaged and their standard deviation provides a measure for the scatter in $\Delta W_{\mathrm{MHD}}$, which will be represented with error bars.

$\Delta W_{\text {TS }}$ is evaluated by applying the method explained in [36]. In the steady phase of the pulse, the pre- and post-ELM TS profiles are fitted using the ELM synchronisation technique to compensate for the low time resolution $(20 \mathrm{~Hz})$ of the TS diagnostic. The post-ELM profile fit represents roughly the 5-15\% interval of the ELM period. TS measurements taken during the ELM crash are excluded as the profiles in this interval are often dominated by the particular dynamics of each ELM crash. Typically 2 or 3 TS measurements following the ELM crash are selected. An example is shown in figure 13, 


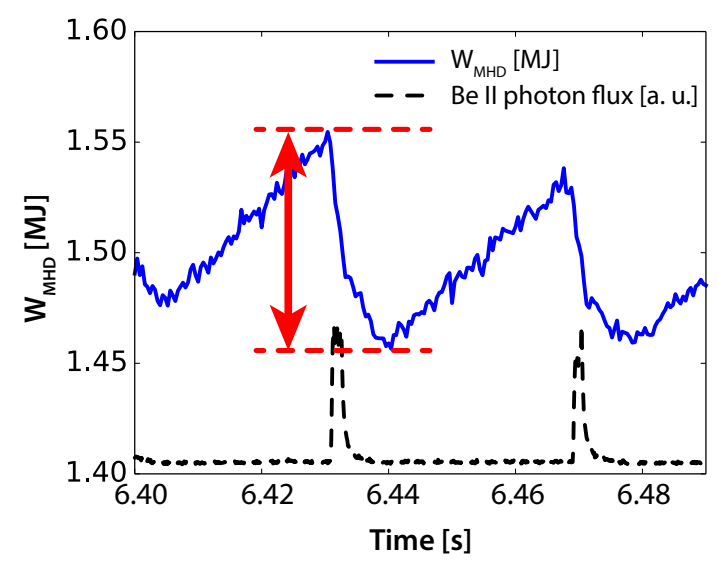

Figure 12. Stored energy signal $\left(W_{\mathrm{MHD}}\right)$ from EFIT equilibrium reconstruction in blue during an ELM crash in H pulse \#91554. The inner divertor BeII $(\lambda=527 \mathrm{~nm})$ photon flux (dashed black line) is used as an ELM marker.

where pre-ELM (80-97 \%) and post-ELM (5-15\%) electron density (figure 13a) and temperature (figure 13b) TS profiles can be seen for D discharge \#84796. The ELM energy loss is calculated from the difference between the pre- and post-ELM TS profiles volume integrated in the region $\Psi_{N}=[0.5,1.05] . T_{i}=T_{e}$ is assumed and line-averaged $Z_{\text {eff }}$ measurements (assuming Be as single impurity) are taken into account to evaluate the total ELM energy loss. Due to ELM synchronisation, $\Delta W_{\mathrm{TS}}$ is already an average over the steady phase of the discharge. The uncertainty of $\Delta W_{\mathrm{TS}}$ is estimated from the errors of the pedestal top values of the mtanh fit.

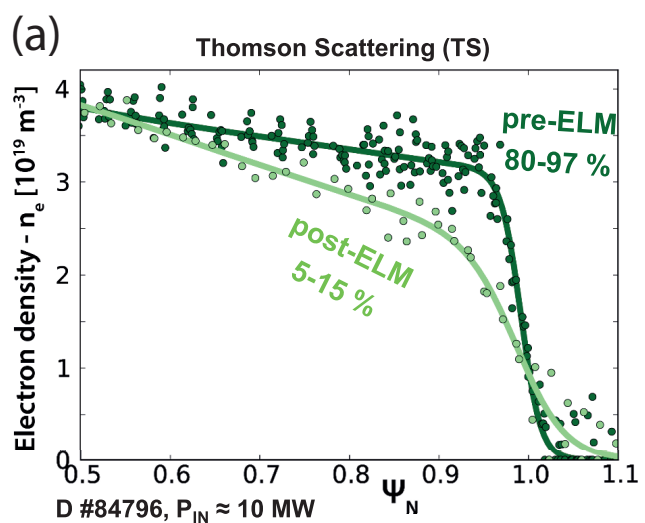

(b)

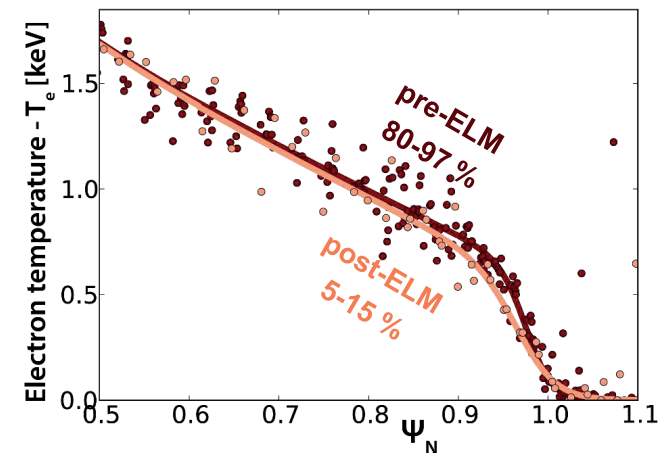

Figure 13. The mtanh fitted pre-ELM (80-97 \%) and post-ELM (5-15\%) density (a) and temperature (b) profiles of D pulse \#84796.

A comparison of ELM losses evaluated from $\Delta W_{\mathrm{MHD}}$ and $\Delta W_{\mathrm{TS}}$ for selected pulses of the isotope dataset is shown in figure 14. The ELM losses evaluated by the two measurements are broadly consistent, especially in controlled parameter scans, but differences between individual discharges can be as high as a factor of 2 due to the intrinsic uncertainties of this analysis. These include uncertainties in $\Delta W_{\text {MHD }}$ due to slow data acquisition of the magnetic diagnostics, screening of the vacuum vessel and 
other conducting structures and uncertainties in $\Delta W_{\mathrm{TS}}$ due to uncertainties in the TS measurement and errors introduced by ELM-synchronisation and regularisation of the profile by the mtanh fit. Due to these difficulties with the measurements, ELM energy losses can only be evaluated typically below $f_{\mathrm{ELM}} \approx 50-60 \mathrm{~Hz}$. Thus, figure 14 shows a subset of JET-ILW H and D type I ELMy H-modes, where the ELM frequency satisfies this condition.

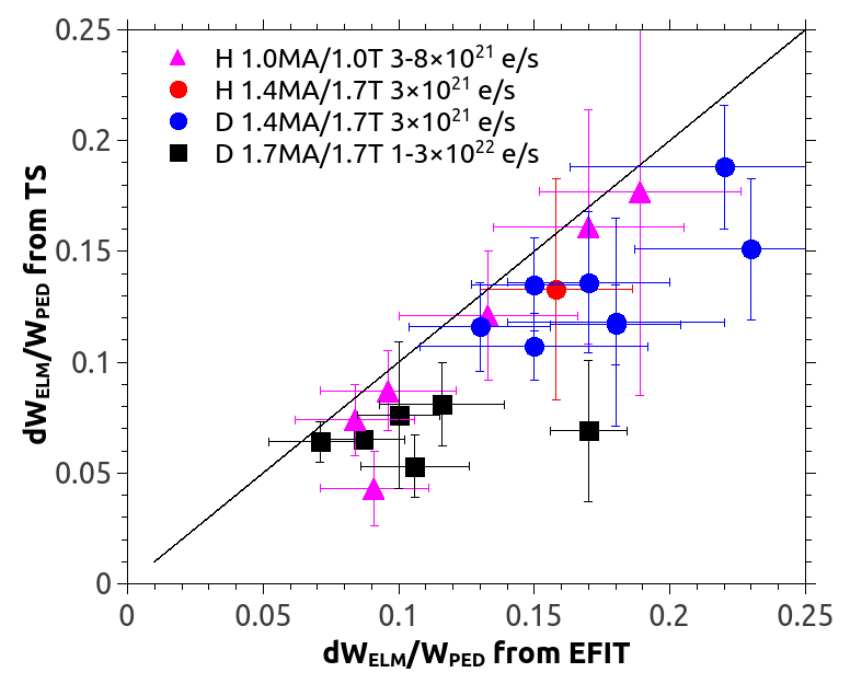

Figure 14. Comparison of ELM losses evaluated from $W_{\mathrm{MHD}}$ and TS profiles on a subset of JET-ILW H and D type I ELMy H-modes.

The power balance analysis is presented here for selected discharges with plasma current $I_{p}=1.4 \mathrm{MA}$, toroidal magnetic field $B_{t}=1.7 \mathrm{~T}$, fuelling gas rate $\Gamma_{e}=$ $3-4 \times 10^{21} \mathrm{e} / \mathrm{s}$ and NBI heating. The ELM-averaged pedestal kinetic profiles of the selected pulses are shown in figure 15. It shows the pedestal profiles of electron density, temperature and pressure for a $\mathrm{H}$ reference discharge in red (\#91554) and two deuterium plasmas with similar thermal stored energy (in blue, \#84793) and input power (in black, \#84796) of the hydrogen counterpart. The main parameters of these 3 discharges can be seen in table 1.

\begin{tabular}{|r|c|c|c|c|c|c|c|c|c|}
\hline Shot & Isotope & $\begin{array}{c}I_{\mathrm{p}} \\
{[\mathrm{MA}]}\end{array}$ & $\begin{array}{c}B_{\mathrm{t}} \\
{[\mathrm{T}]}\end{array}$ & $\begin{array}{c}\Gamma_{\mathrm{D}} \\
{\left[10^{21} \mathrm{e} / \mathrm{s}\right]}\end{array}$ & $\delta$ & $\begin{array}{c}P_{\mathrm{NBI}} \\
{[\mathrm{MW}]}\end{array}$ & $\begin{array}{c}P_{\text {loss }} \\
{[\mathrm{MW}]}\end{array}$ & $\begin{array}{c}W_{\text {th }} \\
{[\mathrm{MJ}]}\end{array}$ & $\begin{array}{c}f_{\text {ELM }} \\
{[\mathrm{Hz}]}\end{array}$ \\
\hline$\# 84793$ & $\mathrm{D}$ & 1.4 & 1.7 & 2.8 & 0.2 & 4.4 & 4.6 & 1.2 & 14.2 \\
$\# 84796$ & $\mathrm{D}$ & 1.4 & 1.7 & 2.8 & 0.2 & 10.6 & 10.4 & 1.9 & 18.7 \\
$\# 91554$ & $\mathrm{H}$ & 1.4 & 1.7 & 4 & 0.2 & 10.0 & 9.4 & 1.1 & 31.3 \\
\hline
\end{tabular}

Table 1. Main parameters of the $\mathrm{H}$ and $\mathrm{D}$ plasmas selected for comparison. $P_{\mathrm{NBI}}$ is the NBI heating power, $P_{\text {loss }}$ is the loss power (i.e. the total absorbed power compensated with the time derivative of the total stored energy), $W_{\mathrm{th}}$ is the thermal stored energy calculated as 2 times the electron stored energy from TS measurements and $f_{\mathrm{ELM}}$ is the ELM frequency. 
The comparison of $\mathrm{H}$ and D plasmas at similar stored energy (\#91554 and \#84793) shows that roughly two times higher heating power is needed in $\mathrm{H}$ to match the stored energy of the D counterpart. In this comparison the pedestal pressure is also similar and the lower density in $\mathrm{H}$ is compensated by the higher temperature as shown in figure 15 . When the heating power is similar in $\mathrm{H}$ and $\mathrm{D}$ (\#91554 and \#84796), the pedestal temperature is similar too, but the pedestal density is significantly lower in $\mathrm{H}$.
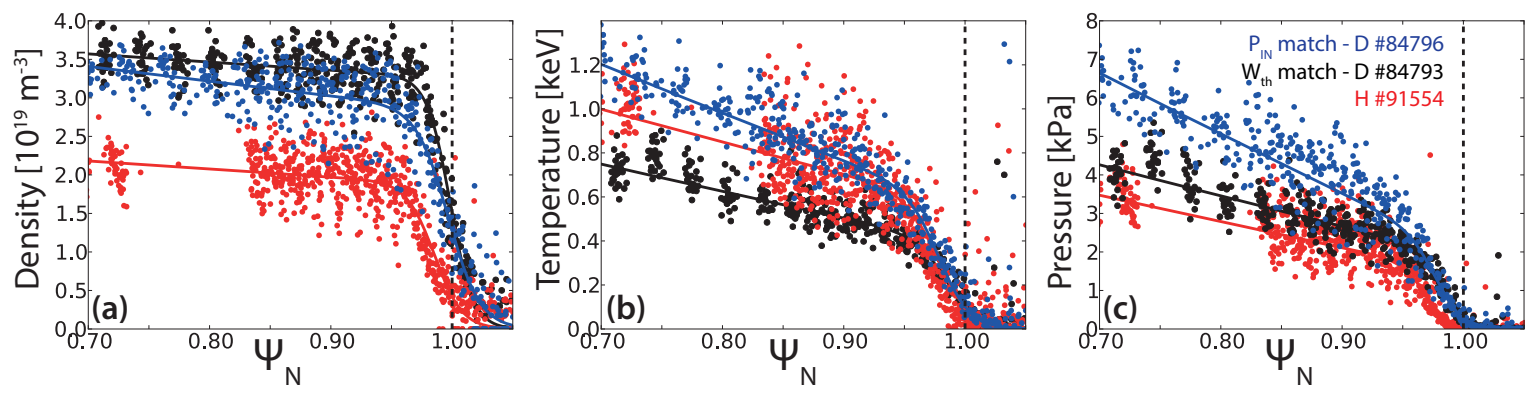

Figure 15. ELM-averaged (0-100\% of the ELM cycle) electron kinetic profiles from TS of the pedestal for the hydrogen reference discharge (\#91554) and the two deuterium plasmas with similar thermal stored energy (\#84793) and similar input power (\#84796) to that of the hydrogen counterpart. (a) Electron density (b) electron temperature (c) electron pressure. These profiles are radially shifted to have $T_{\mathrm{e}, \mathrm{sep}}=100 \mathrm{eV}$.

The result of the power balance analysis for the 3 discharges characterised above is summarised in table 2. The comparison of $\mathrm{H}$ and $\mathrm{D}$ plasmas at similar stored energy (\#91554 and \#84793) shows that roughly double inter-ELM separatrix loss power is required in $\mathrm{H}$ than in $\mathrm{D}$ to maintain the same pedestal top pressure. This is in agreement with observations of ASDEX-Upgrade H-mode plasmas [10]. When the heating power is similar in H and D (\#91554 and \#84796), inter-ELM separatrix loss powers are also similar.

\begin{tabular}{|c|c|c|c|c|c|c|}
\hline Shot & Isotope & $\begin{array}{c}P_{\text {loss }} \\
{[\mathrm{MW}]}\end{array}$ & $\begin{array}{c}P_{\text {rad }} \\
{[\mathrm{MW}]}\end{array}$ & $\begin{array}{c}P_{E L M} \\
{[\mathrm{MW}]}\end{array}$ & $\begin{array}{c}P_{\text {sep }} \text { inter-ELM } \\
{[\mathrm{MW}]}\end{array}$ & $\begin{array}{c}p_{e, P E D} \\
{[\mathrm{kPa}]}\end{array}$ \\
\hline$\# 84793$ & $\mathrm{D}$ & 4.6 & 1.1 & 1.1 & 2.4 & 2.2 \\
$\# 84796$ & $\mathrm{D}$ & 10.4 & 2.2 & 2.4 & 5.8 & 3.1 \\
$\# 91554$ & $\mathrm{H}$ & 9.4 & 2.0 & 2.3 & 5.1 & 2.1 \\
\hline
\end{tabular}

Table 2. Power balance analysis for the three discharges at $1.4 \mathrm{MA} / 1.7 \mathrm{~T}$ and low gas rate introduced in table 1 .

\subsection{ELM particle losses}

We now analyse how ELMs affect the particle and energy channels, respectively. Figure $13 \mathrm{a}$ and $\mathrm{b}$ show the pre-ELM (80-97 \%) and post-ELM (5-15\%) TS profiles 
for discharge \#84796. In this example, the ELMs primarily affect the density, not so much the temperature profiles. This behaviour is general in the analysed dataset and applies to both $\mathrm{H}$ and $\mathrm{D}$ plasmas as indicated by figure 16, where the relative drop of the pedestal top density and temperature is shown for $\mathrm{H}$ and $\mathrm{D}$ plasmas at $1.4 \mathrm{MA} / 1.7 \mathrm{~T}$ (circles) and 1.0 MA/1.0 T (triangles) plasmas. A comparison of the fuelling sources in representative plasmas (from EDGE2D-EIRENE simulations, see section 4) and an order of magnitude estimate for the ELM particle losses utilising the TS measurement suggests that ELMs could be responsible for $\sim 20-30 \%$ of the total particle losses. In view of this, ELM particle losses are investigated here in detail.

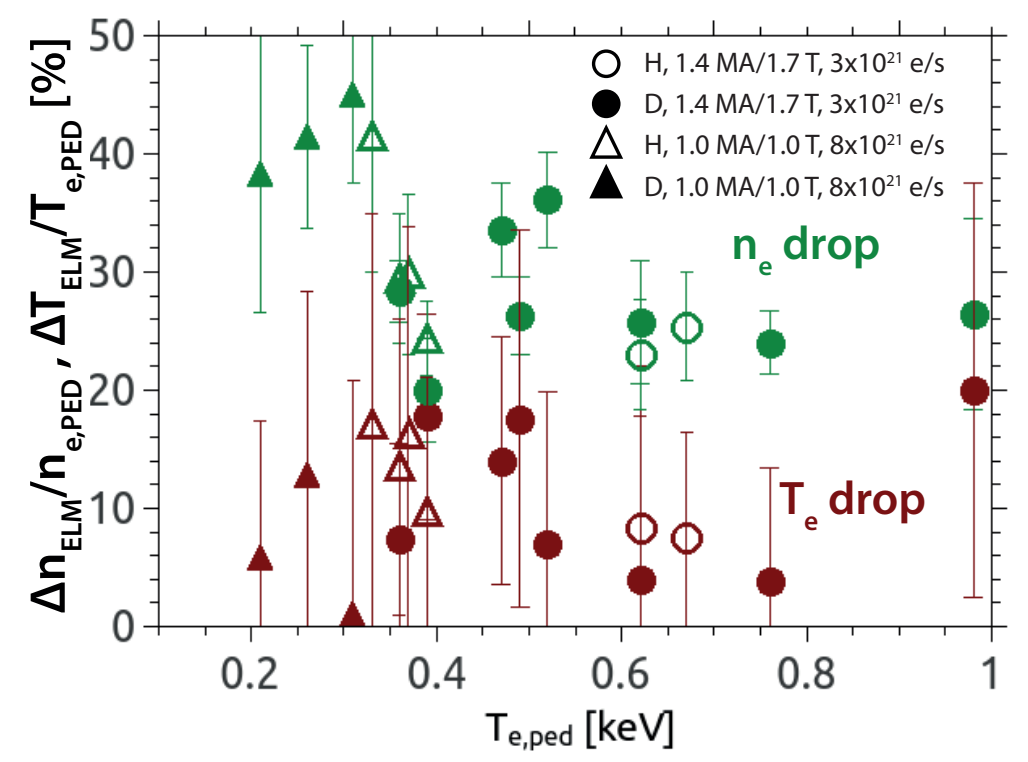

Figure 16. The relative drop of the pedestal top density and temperature during ELMs for $\mathrm{H}$ (open symbols) and D (full symbols) plasmas at $1.4 \mathrm{MA} / 1.7 \mathrm{~T}$ low gas rate (circles) and 1.0 MA/1.0 T medium gas rate (triangles).

ELM energy losses primarily due to the loss of particles have also been observed in low triangularity JET-C experiments, where $f_{\text {ELM }}$ was increased by increasing the fuelling gas rate $[37,38]$. At low $f_{\mathrm{ELM}}$, ELM energy losses (relative to the total stored energy) and the relative temperature drop were high, with smaller relative density drop. With increasing $f_{\mathrm{ELM}}$, the ELM energy losses decreased, primarily due to the reduction of the relative temperature drop and no change in the relative density drop, leading to ELMs mainly affecting the density pedestal, similarly to the ELMs observed in the JET-ILW isotope experiments described here.

As it was shown in section 2 , the pedestal density in $\mathrm{D}$ is significantly higher than in $\mathrm{H}$ at similar $P_{\text {sep }}$ in the JET-ILW isotope dataset. However, it has also been shown that the ELM frequency is typically higher in $\mathrm{H}$ in these conditions. Figure 17a shows the pedestal top density as a function of $f_{\mathrm{ELM}}$ in $\mathrm{H}$ and $\mathrm{D}$ for the low gas $1.4 \mathrm{MA} / 1.7 \mathrm{~T}$ and the medium gas 1.0 MA/1.0 T datasets. In these low triangularity plasmas $(\delta \approx 0.2)$, 
$n_{\mathrm{e}, \mathrm{PED}}$ decreases as $f_{\mathrm{ELM}}$ is increased by increasing input power [22]. $\mathrm{H}$ and D pedestals at similar $f_{\text {ELM }}$ (obtained at different $P_{\text {sep }}$ ) have comparable pedestal densities, as highlighted by the black dashed circles in figure 17a. This suggests that the higher ELM frequency in $\mathrm{H}$ may also play a role in setting the observed lower $n_{\mathrm{e}, \mathrm{PED}}$.
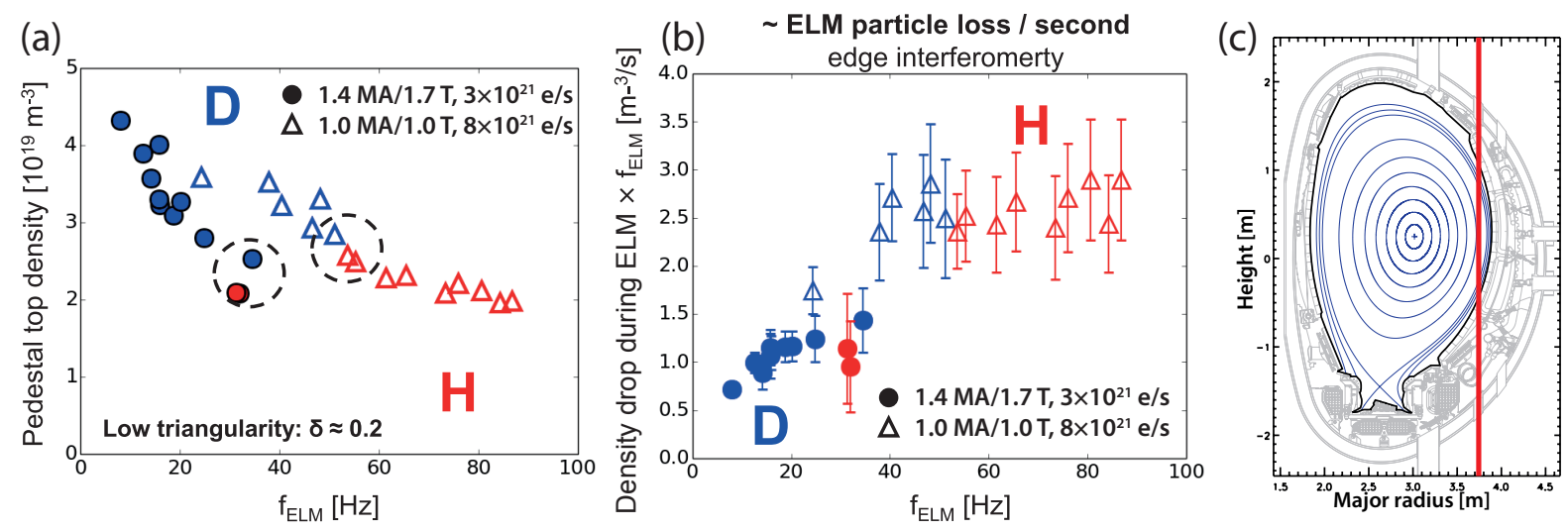

Figure 17. (a) Pedestal top density as a function of $f_{\mathrm{ELM}}$ in low $\delta(=0.2) \mathrm{H}$ and $\mathrm{D}$ plasmas at $1.4 \mathrm{MA} / 1.7 \mathrm{~T}$ (full circles) and 1.0 MA/1.0 T (open triangles). $\mathrm{H}$ and D pedestals at similar $f_{\text {ELM }}$ (but different $P_{\text {sep }}$ ) have comparable pedestal densities as highlighted by the black dashed circles. (b) Average drop in edge interferometer signal during ELMs per second. Deuterium pulses are indicated in blue, hydrogen pulses in red. The error bars represent the statistical variation of the ELM particle loss throughout the steady phase of the discharge. (c) The line-of-sight of the edge interferometry channel (in red) used for the ELM particle loss analysis in a poloidal cross-section of JET-ILW.

The increase of density pump out with increasing $f_{\text {ELM }}$ has been observed in JET experiments utilising ELM pacing via fast vertical plasma motion (vertical kick) [39]. Vertical kicks trigger ELMs by introducing a local perturbation of the current density close to the separatrix. A kick is an intermittent perturbation and only affects the transport by triggering extra ELMs but not modifying the inter-ELM transport [39]. It has been reported that the correlation between the density pump-out and $f_{\text {ELM }}$ suggests that the reduction in the plasma particle content is a consequence of the increase in the time averaged ELM particle loss [39].

In order to understand the cause of the correlation between $f_{\mathrm{ELM}}$ and $n_{\mathrm{e}, \mathrm{PED}}$ in the $\mathrm{H}$ and $\mathrm{D}$ isotope database, the ELM particle losses are investigated in detail. High resolution profiles measurements were not available for the plasmas in the analysed dataset. The TS system has slow time resolution $(20 \mathrm{~Hz})$ and the reflectometry is not available below $B_{t}=2 \mathrm{~T}$. Therefore, the edge interferometer was utilised to estimate the particle loss in an ELM crash. It provides a line integrated density measurement at the plasma edge, as shown in figure 17c. The drop in the line-averaged interferometer signal during the ELM crash times the ELM frequency is taken as proxy for the total ELM induced particle loss, $f_{\mathrm{ELM}} \times \Delta n_{\mathrm{ELM}}$, where $\Delta n_{\mathrm{ELM}}$ is the particle loss caused by the ELM. 
Figure $17 \mathrm{~b}$ shows that at low $f_{\mathrm{ELM}}(<40 \mathrm{~Hz})$ ELM particle losses increase with ELM frequency. This implies that the higher $f_{\mathrm{ELM}}$ in $\mathrm{H}$ than in $\mathrm{D}$ at similar $P_{\text {sep }}$ may contribute to the observed lower pedestal density in H. However, ELM particle losses saturate at higher $f_{\mathrm{ELM}}$, both in $\mathrm{H}$ and in $\mathrm{D}$, indicating that other mechanisms may also play a role in setting lower density in H. EDGE2D-EIRENE simulations discussed in the next section indicate that higher particle transport (ELM and inter ELM particle transport together) in $\mathrm{H}$ than in $\mathrm{D}$ is likely to play an important role in the observed lower pedestal density in $\mathrm{H}$.

\section{Interpretative EDGE2D-EIRENE simulations}

In the present section the plasma edge properties of $\mathrm{H}$ and $\mathrm{D} \mathrm{H}$-modes are investigated using interpretative EDGE2D-EIRENE simulations [16, 17, 18]. EDGE2D is a 2D fluid code with realistic geometry of the SOL and divertor region, which is coupled to EIRENE, a Monte Carlo code used to calculate the neutral particle distribution. The two-dimensional edge transport simulations are also used here to obtain a more accurate value for the electron separatrix temperature than the typically used $T_{\mathrm{e} \text {,sep }} \approx 100 \mathrm{eV}$ obtained by the 2-point model $[40,41]$. A constraint on $T_{e, s e p}$ is required due to the uncertainties in the EFIT magnetic equilibrium reconstruction, in particular for the separatrix position, which makes the radial alignment of the measured kinetic profiles inaccurate. An estimate for $T_{e, s e p}$ can be obtained by taking into account the parallel heat conduction and pressure and power balance in the scrape-of-layer (SOL), thus obtaining a relation between the upstream $\left(T_{e \text {,upstream }} \equiv T_{e, s e p}\right)$ and divertor target temperatures $\left(T_{e, \text { target }}\right)$. Assuming a conduction limited divertor regime, the upstream temperature at the separatrix can be approximated by the two point model equation [41]:

$$
T_{e, \text { upstream }}=\left(T_{e, \text { target }}^{7 / 2}+\frac{7 P_{s e p} L}{2 A_{q} \kappa}\right)^{\frac{2}{7}},
$$

where $L$ is the connection length, $A_{q}$ is the projection of the wetted area perpendicular to the heat flux and $\kappa$ is the parallel heat conductivity coefficient. $T_{e, \text { target }}$ is often neglected as $T_{e, \text { upstream }}^{7 / 2} \gg T_{e, \text { target }}^{7 / 2}$ is satisfied in a conduction limited divertor regime. It is common practice to assume that in eq. (5) $P_{\text {sep }}$ is the only significantly varying parameter and

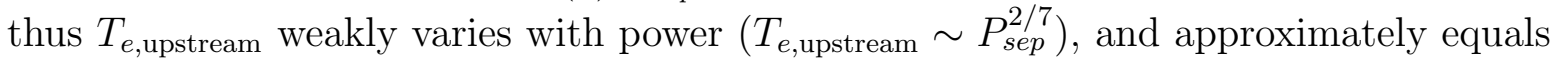
$100 \mathrm{eV}$ for JET H-modes. However, $A_{q}$ could also change from discharge to discharge and the condition of the conduction limited divertor regime is not necessarily satisfied in all cases. In the sheath limited regime $T_{e, \text { target }}^{7 / 2}$ cannot be neglected. Thus, in realistic experimental conditions, $T_{\mathrm{e} \text {,sep }}$ could vary from $100 \mathrm{eV}$ and in extreme cases it might have a significant effect on pedestal stability $[42,43]$.

A more accurate estimate for $T_{e, s e p}$ can be gained by using $2 \mathrm{D}$ SOL transport codes. Interpretative EDGE2D-EIRENE simulations indicate a higher ion and electron temperature at the separatrix in $\mathrm{H}$ than in $\mathrm{D}$ in the pair of type I ELMy H-modes at low gas rate (\#91554 and \#84793) with similar stored energy, but lower $n_{\mathrm{e}, \mathrm{PED}}$ and higher $T_{\mathrm{e}, \mathrm{PED}}$ in $\mathrm{H}$ and $\mathrm{D}$ (see figure 19). Note that the input power is doubled in $\mathrm{H}$ compared 
to $\mathrm{D}$ to reach the same stored energy and pedestal pressure. In these interpretative runs the perpendicular transport coefficient of electron particle diffusion $D_{\perp}\left(\Gamma_{e}=D_{\perp} \nabla n_{e}\right)$, electron and ion heat transport $\chi_{e, i}\left(q_{e, i}=-n_{e, i} \chi_{e, i} \nabla T_{e, i}\right)$ and the pump albedo were iterated until the solution fitted the upstream $n_{e}$ and $T_{e}$ profiles (measured by TS) and the outer target heat deposition profile (measured by IR-camera). $\chi_{e}$ and $\chi_{i}$ were assumed to be the same. $\chi$ in the SOL was set such that the heat deposition profile at the outer divertor target matched the IR camera measurements. The aim here was to approximately match the width of the heat deposition profile in order to constrain $A_{q}$, which potentially affects $T_{e, s e p}$ as it is implied by the two-point model in eq. (5). Cross-field drifts were not taken into account in the simulations.

The grid for EDGE2D relies on EFIT equilibrium reconstruction and is shown in cyan in figure 18a. The EDGE2D simulation domain extends to $\sim 10-15 \mathrm{~cm}$ inside the separatrix to the core plasma. The input power in EDGE2D was set to the power crossing the separatrix inter-ELM, i.e. the ELM power loss $\left(P_{\text {ELM }}\right)$ was excluded. The ELM crash was not simulated and it was assumed that $P_{\text {ELM }}$ does not contribute to the power balance in the SOL, which sets the separatrix temperature inter-ELM. For simplicity, both the H (\#91554) and the D (\#84793) case were simulated in cornercorner $(\mathrm{C} / \mathrm{C})$ divertor target configuration, albeit discharge \#84793 was in verticalhorizontal $(\mathrm{V} / \mathrm{H})$ divertor target configuration in the experiment. The wall structure had to be slightly modified around the outer strike-point so that the EDGE2D grid does not cross wall surfaces as shown in figure 18b. The real divertor geometry (in green) is slightly altered to be aligned with the outermost grid elements. This modification could possibly lead to differences in the neutral pressure around the outer strike point, but the aim here was to compare a pair of $\mathrm{H}$ and $\mathrm{D}$ simulations and not a comprehensive validation of the code. Thus, both the $\mathrm{H}$ and the $\mathrm{D}$ cases were simulated using the same grid. Quantitative answer on how much the wall structure alteration affects the target and upstream profiles could only be given by testing this effect with an edge transport code such as SolEdge2D [44, 45] that is capable of handling complex geometries, but this is out of the scope of the present work. As it was explained in section 2, pumping is more effective in $\mathrm{C} / \mathrm{C}$ configuration, thus the pump albedo is likely to be overestimated in our EDGE2D-EIRENE simulations for the $\mathrm{D}$ case. The gas fuelling was set in accordance with the experiment. The effect of divertor configuration $(\mathrm{C} / \mathrm{C}$ vs $\mathrm{V} / \mathrm{H})$ on the pedestal density has been investigated in a separate study, where all input parameters of the interpretative simulation for \#84793 (transport coefficients, pump albedo, input power, gas fuelling, etc.) were kept fixed, but the divertor configuration was changed from $\mathrm{C} / \mathrm{C}$ to $\mathrm{V} / \mathrm{H}$. The pedestal density increased by approximately $15-20 \%$, while $T_{\mathrm{e}, \mathrm{PED}}$ decreased leading to virtually no change in pedestal pressure, which is consistent with the experimental observations.

Figure 19 shows the "inter-ELM" (40-80\% of the ELM cycle) $n_{e}$ and $T_{e}$ profiles measured by TS (in grey) and the profiles of the EDGE2D-EIRENE solutions (in red for $\mathrm{H}$ and blue for $\mathrm{D}$ ). The "inter-ELM" outer target heat flux profiles are evaluated by averaging the profiles from the IR camera in the 40-80\% part of the ELM cycle and are 

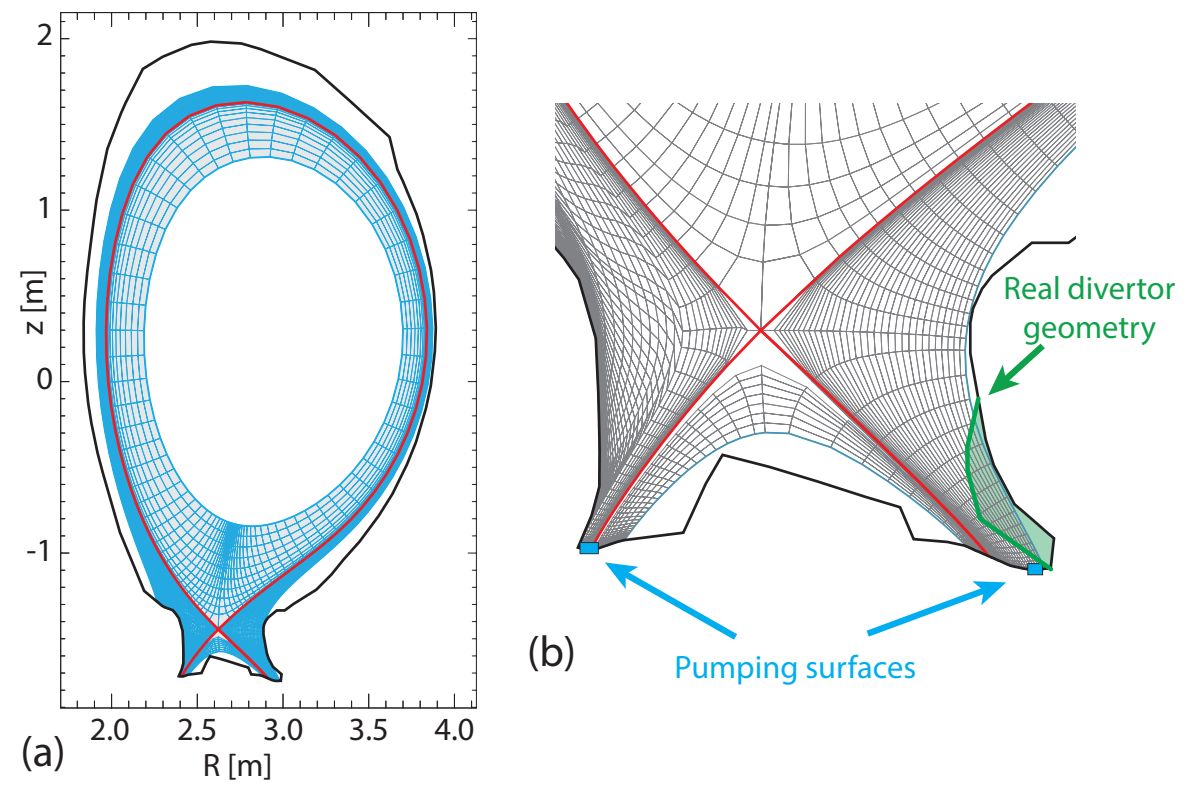

Figure 18. (a) The EDGE2D simulation grid in cyan. (b) The real divertor geometry (in green) is slightly altered at the outer strike point to be aligned with the outermost grid elements. The pump surfaces are shown in cyan close to the strike points.

compared with the EDGE2D simulations in figure 20. The width of the heat deposition profile is similar in $\mathrm{H}$ and $\mathrm{D}$, thus a difference in $T_{e, s e p}$ between the two cases due to different $A_{q}$ is not expected. The time evolution of heat deposition reconstructed from IR measurements can be very uncertain in the vicinity of the ELM crash due to the transient heat flux arriving to the target. Thus, the 40-80\% interval was chosen to represent the inter-ELM parameters and exclude any artefacts in the heat deposition profiles. TS data are also filtered for the 40-80 \% part of the ELM cycle for consistency. Figure 21 shows the anomalous transport coefficients used in the simulations to match the experimental profiles. The anomalous heat transport coefficients inside of the pedestal are higher in $\mathrm{H}$ than in $\mathrm{D}$, but are comparable in the edge transport barrier (ETB). $D_{\perp}$ is higher in $\mathrm{H}$ than in $\mathrm{D}$ everywhere inside the separatrix. This implies that larger particle transport in $\mathrm{H}$ than in $\mathrm{D}$ could be responsible for the different pedestals, which is consistent with the experimental observations suggesting higher ELM particle losses in $\mathrm{H}$ than in $\mathrm{D}$ as discussed in section 3. Note that in the EDGE2D-EIRENE simulations $D_{\perp}$ represents the total particle transport, which - in the experiment - is a sum of the ELM and the inter-ELM particle losses. The "steps" in $D_{\perp}$ and $\chi_{e, i}$ in the ETB (see figure 21) were needed in EDGE2D-EIRENE to match the TS data as close as possible, but it is important to note that $T_{\mathrm{e}, \mathrm{sep}}$ is not sensitive to these fine details in the shape of the perpendicular transport coefficients. $\chi_{e, i}$ outside the separatrix is constrained by the outer target heat deposition profile, but there is insufficient information to distinguish between H and D. Similarly, there is no available density data outside the separatrix to constrain $D_{\perp}$, which was therefore chosen to be the same in $\mathrm{H}$ and $\mathrm{D}$. It is important to note that a particle pinch, which may have an important role in the 
particle transport $[46,47,48]$ is not introduced in these simulations. The experimental $n_{e}$ profile shape could be reproduced with different variations of the diffusion coefficient and the pinch velocity, due to the lack of constraints. The particle pinch is not taken into account in these EDGE2D-EIRENE simulations, but its role in edge particle transport is not excluded and is the subject of ongoing research.
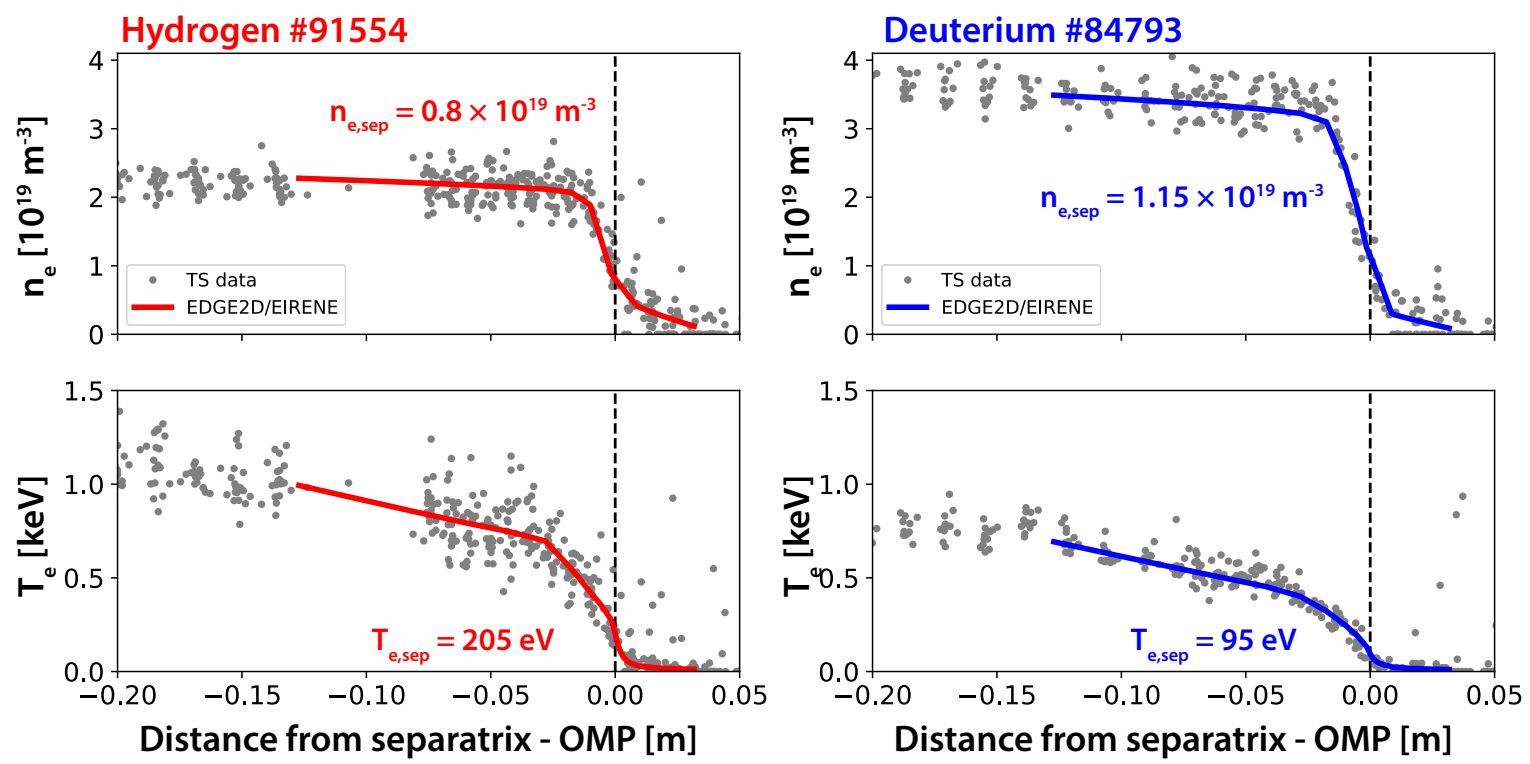

Figure 19. Inter-ELM TS profiles (40-80\% of the ELM cycle) for $n_{e}$ and $T_{e}$ (in grey) in the steady phase of the $\mathrm{H}$ (\#91554: 5.7-8.2 s) and $\mathrm{D}$ (\#84793: 5.0-6.3 s) discharges. The resulting upstream $n_{e}$ and $T_{e}$ profiles of the interpretative EDGE2D-EIRENE simulations for the H pulse (\#91554) in red and for the D pulse (\#84793) in blue.

As highlighted in figure 19, the EDGE2D-EIRENE simulations indicate that $T_{\mathrm{e}, \text { sep }}$ is higher in the $\mathrm{H}$ discharge $(\approx 205 \mathrm{eV})$ than in the $\mathrm{D}(\approx 95 \mathrm{eV})$. This difference is due to the fact that roughly two times more power crosses the separatrix at lower density in the $\mathrm{H}$ case. As a result, the $\mathrm{H}$ discharge in the EDGE2D simulation is found to be clearly in the sheath limited regime with $T_{\mathrm{e} \text {,upstream }} \approx 205 \mathrm{eV}$ and $T_{\mathrm{e} \text {, target }} \approx 200 \mathrm{eV}$. In contrast, for the $\mathrm{D}$ case $T_{\mathrm{e} \text {,upstream }} \approx 95 \mathrm{eV}$ and $T_{\mathrm{e} \text {,target }} \approx 75 \mathrm{eV}$. These are still high temperatures at the divertor target, but the temperature drop between upstream and target is larger in the $\mathrm{D}$ case than in $\mathrm{H}$. Therefore, the $\mathrm{D}$ discharge is closer to the conduction limited regime than the $\mathrm{H}$ discharge.

The outer divertor target $T_{e}$ obtained in the EDGE2D-EIRENE simulation for the D case is supported by Langmuir probe measurements, as shown in figure 22 . Note that these high $T_{\text {e,target }}$ values are not typical of JET-ILW operation but of the low gas fuelling of this discharge. In the $\mathrm{H}$ pulse (\#91554), due to lack of Langmuir probe measurements, the high $T_{\text {e,target }}$ suggested by the EDGE2D simulation cannot be compared with experiment. Note that at such high divertor target temperature, above $100 \mathrm{eV}$ as indicated by EDGE2D-EIRENE simulations in H, secondary electron emission could also be important. However, this effect, which would act as an extra electron heat 


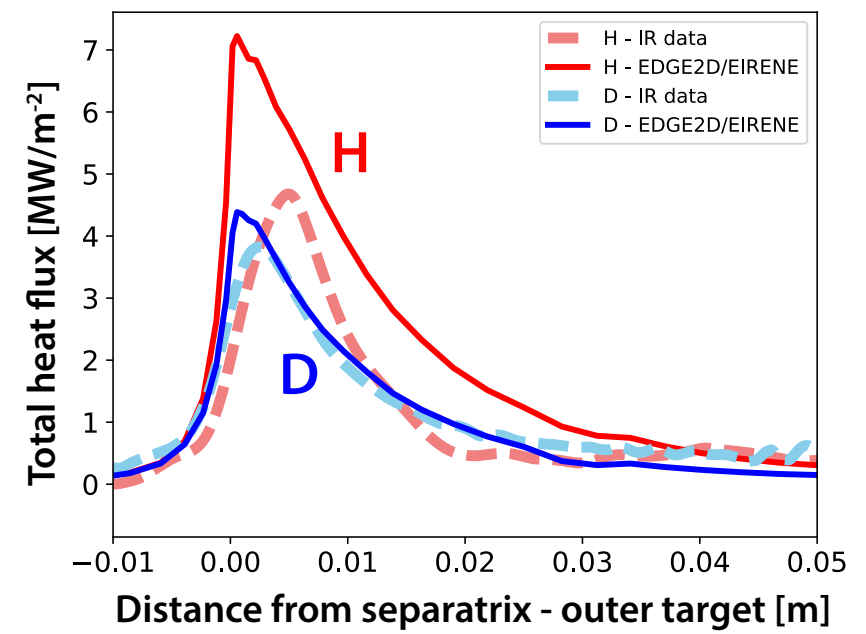

Figure 20. Inter-ELM (40-80 \% of the ELM cycle) outer target heat deposition profiles as evaluated from IR camera measurements in the steady phase of the $\mathrm{H}$ (\#91554: 5.78.2 s) and D (\#84793: 5.0-6.3 s) discharges with dashed lines. Outer divertor target heat deposition profiles from EDGE2D-EIRENE with solid lines for $\mathrm{H}$ (red \#91554) and D (blue \#84793).
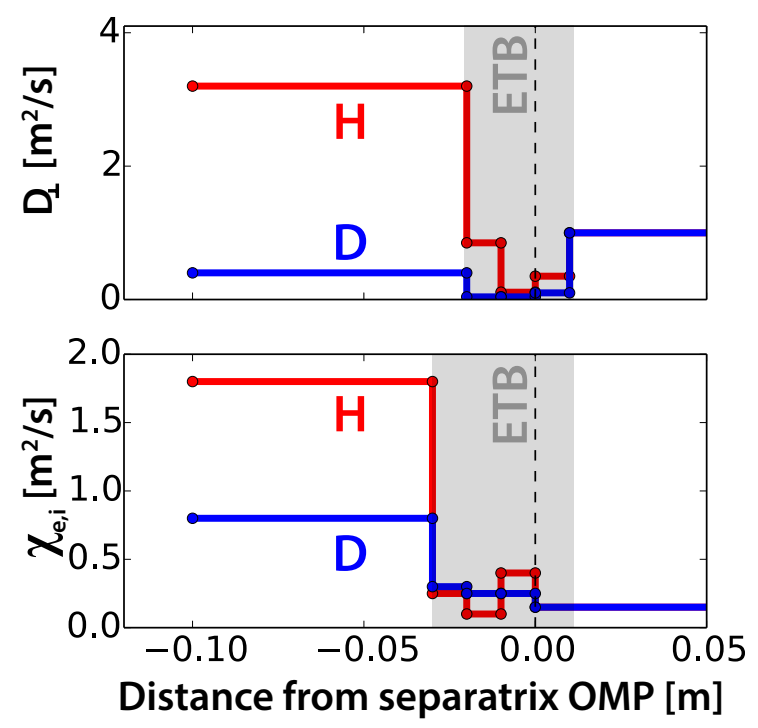

Figure 21. Electron particle diffusion $\left(D_{\perp}\right)$ and electron and ion heat transport $\left(\chi_{e}=\chi_{i}=\chi_{e, i}\right)$ coefficients of the EDGE2D-EIRENE simulations for $\mathrm{H}$ (red \#91554) and D (blue \#84793). The edge transport barrier (ETB) is indicated by the grey shaded area.

sink in the SOL, is not included in the EDGE2D model. Thus, the obtained high $T_{\text {esep }}=205 \mathrm{eV}$ for the $\mathrm{H}$ discharge should only be taken as an absolute upper limit and must not be considered at face value.

In the simulations, the pumping surfaces were placed at the corners of the simulated divertor (see figure 18b), where - in experiment - neutrals would be moving into the subdivertor region by the action of the cryopump. The pump efficiency is defined by the pump albedo, which gives the probability that a neutral - which reaches the pumping 


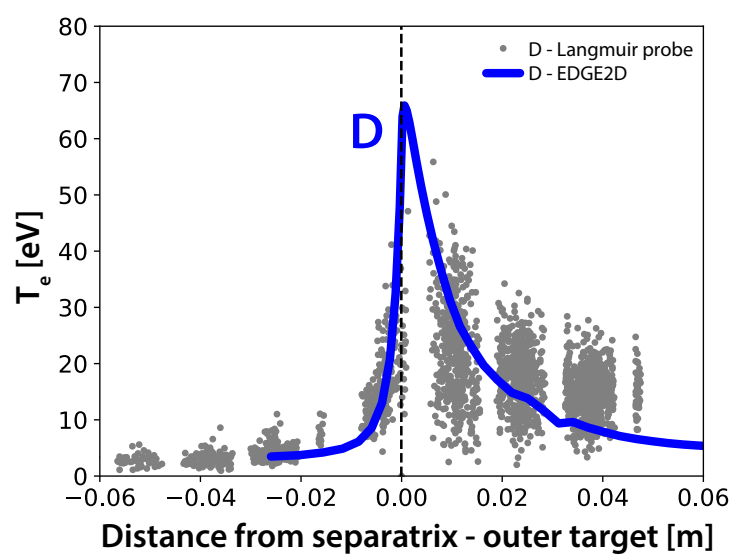

Figure 22. Inter-ELM (40-80\%) outer target $T_{e}$ profile as evaluated from Langmuir probe measurements for the steady phase of the D pulse \#84793 (5.0-6.3 s) in grey and $T_{e}$ from EDGE2D-EIRENE in blue.

surface - is reflected. The pump albedo was set to 0.4 for $\mathrm{H}$ and to 0.7 for $\mathrm{D}$ to obtain a match of the upstream profiles. However, these values are not consistent with testbed results on the sticking coefficients $\left(\alpha_{s}\right)$ of $H_{2}$ and $D_{2}$ at a cryopump surface $[49,50]$. The sticking coefficient is the ratio of the number of particles sticking to the cryosurface related to the total number of particles impinging on it. The sub-divertor structures and the cryopump is not modelled in these EIRENE simulations, thus one-to-one comparison between the albedo defined in EDGE2D-EIRENE and the testbed results for the sticking coefficients on a cryosurface is not possible, but the albedo is roughly proportional to $\left(1-\alpha_{s}\right)$. The testbed results show that the sticking coefficient is higher in D than in $\mathrm{H}[49,50]$, implying lower albedo in $\mathrm{D}$ than in $\mathrm{H}$ in contrast to the EDGE2D-EIRENE pump albedo settings. A possible reason for this disagreement could be that the much hotter ions, electrons and neutrals in the SOL of the $\mathrm{H}$ discharge may have resulted in different neutral recycling at the wall.

The effect of change in mean free path of neutrals between $\mathrm{H}$ and $\mathrm{D}$ in the EDGE2D-EIRENE simulations is investigated with a numerical experiment where all input parameters (transport coefficients, pump albedo, input power, gas fuelling, etc.) are kept fixed, but only the isotope mass is changed from $\mathrm{D}$ to $\mathrm{H}$. The input parameters of the interpretative simulation for the D case (\#84793) were taken. The results of the change from $\mathrm{D}$ to $\mathrm{H}$ for otherwise fixed conditions is $\mathrm{a} \sim 10 \%$ increase in the pedestal top density and a slight decrease in the temperature as shown in figure 23 . The change in the upstream profile is small and opposite to experimental observations. This result - together with the previous findings that higher transport coefficients are required in $\mathrm{H}$ (\#91554) than in D (\#84793) to match the experimental profiles - indicate that the change in neutral penetration due to different isotope mass does not explain the observed lower density in $\mathrm{H}$ pedestals and transport must also play a role. 


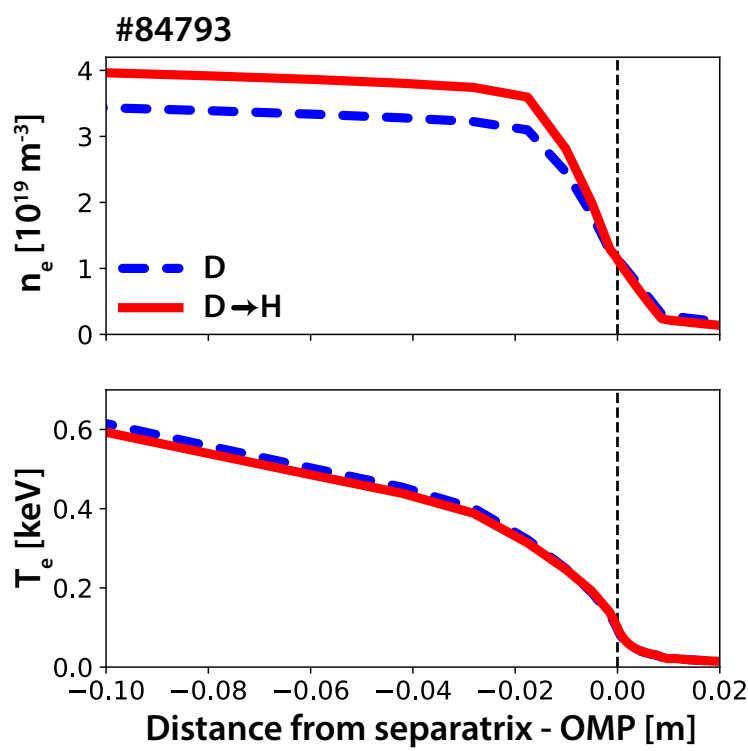

Figure 23. Upstream $n_{e}$ and $T_{e}$ profiles of a pair EDGE2D-EIRENE simulations where all parameters were kept fixed (transport coefficients, pump albedo, input power, gas fuelling, etc.), but the isotope was changed from $\mathrm{D}$ to $\mathrm{H}$.

The interpretative EDGE2D-EIRENE simulations indicate that $T_{\mathrm{e} \text {,sep }}$ is mostly affected by the input power and $\chi_{e, i}$ values in the SOL, which in turn are constrained by the outer target heat flux profile from IR. When both the upstream kinetic profile and outer target heat flux constraints are given, $T_{\mathrm{e} \text {,sep }}$ is a robust parameter in the simulation and is not sensitive to the $D_{\perp}$ and $\chi_{e, i}$ profiles inside the separatrix. The main conclusion of this modelling exercise is that the higher power and lower density in the $\mathrm{H}$ discharge compared to the $\mathrm{D}$ discharge result in a sheath limited divertor regime in the $\mathrm{H}$ case, which leads to high temperature both upstream and at the target, thus suggesting that $T_{\mathrm{e} \text {,sep }}$ in $\mathrm{H}$ is higher than $T_{\mathrm{e}, \mathrm{sep}}$ in $\mathrm{D}$, while $T_{\mathrm{e}, \mathrm{sep}}(D) \approx 100 \mathrm{eV}$ as originally assumed using the 2-point model. This difference in divertor regime between the two cases is a consequence of the density and input power difference in $\mathrm{H}$ and $\mathrm{D}$ and not the isotope mass alone. Due to the limitations of the model as discussed above, the $T_{\text {e,sep }}$ value quoted in figure 19, must not be regarded as the exact solution for the $\mathrm{H}$ case, but as an indication that $T_{\mathrm{e}, \mathrm{sep}}$ could be larger than $100 \mathrm{eV}$ in the $\mathrm{H}$ pulse. Note that the selected pair of discharges are representative of the conditions at low gas fuelling rates. It is expected that at higher gas rate (and density) the divertor regime shifts towards conduction limited regime also in $\mathrm{H}$, where lower target and upstream temperatures are expected than those obtained in the EDGE2D-EIRENE simulations for the $\mathrm{H}$ discharge at low gas rate $(\# 91554)$.

\section{Linear MHD pedestal stability}

A well-known theory which appears to explain the stability conditions of type I ELMy pedestals is the peeling-ballooning (P-B) model [20, 21]. In this model, edge pressure 
gradient and edge current drive coupled P-B modes, which limit the maximum achievable pedestal gradient and trigger an ELM. In this section the pedestal P-B stability of selected $\mathrm{H}$ and $\mathrm{D}$ discharges is examined and the effect of isotope mass on pedestal stability is discussed.

The linear growth rate $\left(\gamma_{\mathrm{MHD}}\right)$ of ideal MHD modes scales as $\sim A^{-1 / 2}$. In numerical stability codes the stability criterion is often set as a small proportion of the Alfvenfrequency $\gamma_{\mathrm{MHD}}>c \times \omega_{A}$, instead of $\gamma_{\mathrm{MHD}}>0 . \quad c$ is typically $\approx 0.02-0.05$ and $\omega_{A}=B_{0} /\left(R_{0} \sqrt{4 \pi \rho_{0}}\right)$ with $\rho_{0}$ the mass density. As $\omega_{A}$ and $\gamma_{\mathrm{MHD}}$ scale with isotope mass in the same way, this stability criterion is independent of $A$.

An isotope dependence of the linear stability is introduced when diamagnetic stabilisation [51] is considered. The diamagnetic drift is expected to stabilise modes - particularly at high toroidal mode number $n$ - when the diamagnetic frequency $\left(\omega_{\text {dia }}\right)$ is comparable to $\gamma_{\mathrm{MHD}}$. $\omega_{\mathrm{dia}}=m / r \times T_{i} /\left(e_{i} B_{0}\right) \times \mathrm{d} \ln p_{i} / \mathrm{d} r$, where $T_{i}, e_{i}$ and $p_{i}$ are temperature, charge, and pressure of the ions, $B_{0}$ is the equilibrium magnetic field, $r$ is the minor radius, and $m$ is the poloidal mode number which is linked to the toroidal mode number $(n)$ via the safety factor $(q): m=n q$. Diamagnetic stabilisation can be taken into account in ideal MHD stability analysis by modifying the stability criterion to $\gamma_{\mathrm{MHD}}>c \times \omega_{\text {dia }}[21,52]$. As $\omega_{\text {dia }}$ is independent of $A$, but $\gamma_{\mathrm{MHD}} \sim A^{-1 / 2}$, larger isotope mass leads to more stable pedestals when this stability criterion is applied.

Figure 24 shows the $j-\alpha$ pedestal stability diagram for the 3 reference discharges of section 3 as calculated with HELENA/ELITE [19, 20, 21]. $j$ is the normalised current density self-consistently calculated with HELENA using Sauter's formula $[53,54]$ for the bootstrap current $\left(j_{\mathrm{BS}}\right)$ and assuming neoclassical resistivity and a fully diffused Ohmic current. Note that Sauter's formula has no isotope mass dependence. $\alpha$ is the normalised pressure gradient as defined in [55]. The inputs for HELENA/ELITE were the fitted kinetic profiles evaluated from Thomson scattering (TS), assuming $T_{e}=T_{i}$ (consistent with charge exchange measurements), line averaged $Z_{\text {eff }}$ with Be as single impurity. The kinetic profiles here are radially aligned so that the separatrix temperature is $100 \mathrm{eV}$. The effect of $T_{\mathrm{e}, \text { sep }}$ on pedestal stability is discussed later in this section.
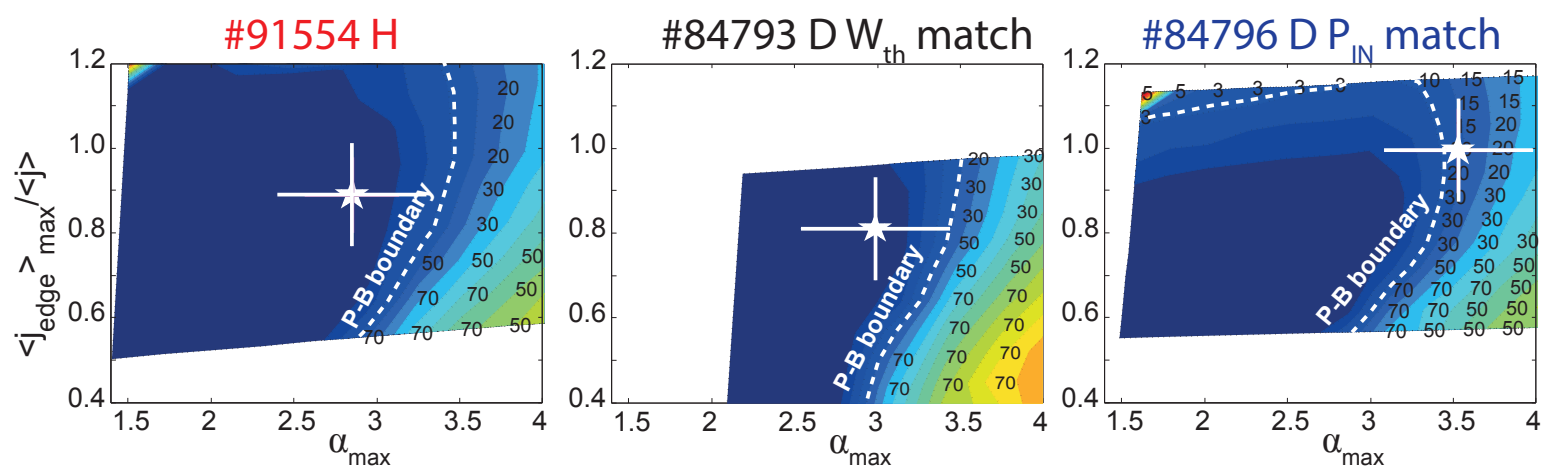

Figure 24. Liner MHD pedestal stability analysis for the hydrogen reference discharge (\#91554) and the two deuterium plasmas matching the total stored energy (\#84793) and the input power $(\# 84796)$ of the hydrogen counterpart. $I_{p}=1.4 \mathrm{MA}, B_{t}=1.7 \mathrm{~T}$. 
In figure 24 , the stability boundary (white dashed lines) is obtained using $\gamma_{\mathrm{MHD}}>$ $0.03 \times \omega_{A}$ stability criterion. The white stars show the operational point of the pedestal as obtained in the experiment. The pedestals in these low gas rate $\left(\Gamma=3 \cdot 10^{21} \mathrm{e} / \mathrm{s}\right) \mathrm{H}$ and D plasmas are close to the P-B boundary within the uncertainties of the operational point. This observation is confirmed with the same analysis performed on a wider dataset, although $\mathrm{H}$ pedestals tend to be on the stable side of the stability boundary. At medium and high gas rate, the operational point moves to the stable region in $\mathrm{H}$, which is similar to what has been found in D at high input power [22]. Pedestals of the 1.0 MA/1.0 T dataset at medium gas are also stable to $\mathrm{P}-\mathrm{B}$ modes both in $\mathrm{H}$ and $\mathrm{D}$, especially at higher power.

The effect of diamagnetic stabilisation is investigated by performing linear ideal MHD stability analysis with HELENA/ELITE on the hydrogen pedestal. Figure 25 shows the $j$ - $\alpha$ stability diagram for $\mathrm{H}$ shot \#91554 profiles using $\gamma_{\mathrm{MHD}}>0.5 \times \omega_{\text {dia }}$ as stability criterion, assuming $A=2$ (dashed blue line) and $A=1$ (solid red line) isotope. When diamagnetic stabilisation is taken into account, the stable region shrinks from $A=2$ to $A=1$ in the calculations indicating less stable pedestals. The difference between the stability boundaries when the isotope is mass is changed from $A=2$ to $A=1$ for discharge $\# 91554$ translates to $\approx 4 \%$ reduction in the critical pedestal pressure height. The critical pedestal pressure height is evaluated by scaling up and down the experimental pressure profile and calculating the associated current profile self-consistently. The pressure profile which is closest to marginal stability gives the critical pressure gradient. Thus, the isotope dependence of linear MHD stability is small and alone does not explain the higher pedestal pressure observed in D type I ELMy H-modes in JET-ILW.

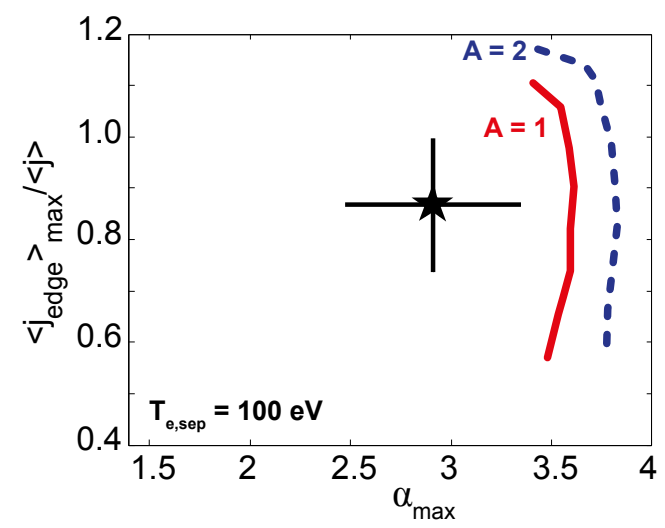

Figure 25. P-B stability of the pre-ELM pedestal of H type-I ELMy H-mode \#91554 as calculated with HELENA/ELITE with the stability criterion $\gamma_{\mathrm{MHD}}>0.25 \times \omega_{\text {dia }}$. The black star indicates the operational point. The blue dashed line shows the stability boundary assuming $A=2$. The stable region shrinks when the isotope mass is changed from D (blue dashed line) to $\mathrm{H}$ (red solid line). The kinetic profiles are radially aligned so that $T_{e, s e p}=100 \mathrm{eV}$. 
The effect of diamagnetic stabilisation in JET pedestals has already been demonstrated using a more rigorous treatment of the diamagnetic drift with an extended MHD model [56, 57]. This approach could be the subject of further studies, however, the results of ideal MHD based estimate used in the present work suggests that only a weak isotope dependence is expected. The effect of sheared rotation on pedestal stability [58] is not discussed here, but it may lead to differences in the pedestal stability between $\mathrm{H}$ and D plasmas as for example the different input power to achieve the same pedestal pressure in $\mathrm{H}$ and D (see section 2) could lead to differences in the NBI torque and thus in the sheared rotation.

While so far we have investigated the pedestal stability assuming $T_{\mathrm{e}, \mathrm{sep}}=100 \mathrm{eV}$ for both $\mathrm{H}$ and $\mathrm{D}$ cases, we now assess the effect of a potentially higher $T_{e, s e p}$ in $\mathrm{H}$ on pedestal stability as suggested by EDGE2D-EIRENE simulations presented in section 4. The linear ideal MHD stability analysis for the hydrogen pulse \#91554 is shown in figure 26, assuming $T_{\mathrm{e}, \mathrm{sep}}=100 \mathrm{eV}$ (solid black), $T_{\mathrm{e}, \mathrm{sep}}=150 \mathrm{eV}$ (dotted green) and $T_{\text {esep }}=200 \mathrm{eV}$ (dashed magenta) as a sensitivity test. The difference in boundary condition at the separatrix translates into significant differences in the P-B stability boundary, with both ballooning and peeling boundaries shrinking due to destabilisation of P-B modes as $T_{\text {e,sep }}$ is increased, similarly to the analysis reported in [43]. The higher $T_{e, s e p}$ shifts the maximum pressure gradient closer to the separatrix, thus it moves to a region of higher magnetic shear, which leads to destabilisation of ballooning modes. At the same time, the edge current profile - which is dominated by the bootstrap current - is also shifted radially outward, leading to higher current at the separatrix, which destabilises peeling modes. The difference in the critical pedestal pressure height between $T_{e, s e p} \approx 100 \mathrm{eV}$ and $200 \mathrm{eV}$ cases is approximately $15 \%$. This change is qualitatively consistent with type I ELMs being triggered at lower pedestal densities in the $\mathrm{H}$ case. As described in section 4, however, such high $T_{\mathrm{e} \text {,sep }}$ in the $\mathrm{H}$ discharge is unrealistic, but the qualitative picture illustrated here warrants further investigation and direct measurement of $T_{\text {e,sep }}$ in $\mathrm{H}$ and in $\mathrm{D}$.

\section{Conclusions and outlook}

In JET-ILW Hydrogen and Deuterium type I ELMy H-mode plasmas a favourable isotope scaling of the thermal energy confinement is observed and the isotope effect originates at the pedestal [1]. In the present paper, the pedestal structure, linear MHD stability and ELM losses have been analysed to gain insight on the dependence of JETILW type I ELMy pedestals on isotope mass.

The pedestal pressure is typically reduced in $\mathrm{H}$ compared to $\mathrm{D}$ at the same input power and gas rate, primarily due to lower pedestal density in $\mathrm{H}$. The pedestal electron pressure gradient is typically lower in $\mathrm{H}$ than in $\mathrm{D}$ at similar pedestal pressure widths. The pedestal density width is typically narrower in $\mathrm{H}$ than in $\mathrm{D}$, which is in contradiction to the neutral penetration model and implies that transport also plays a crucial role in setting the density pedestal and/or that the assumptions of the NPM on neutral 


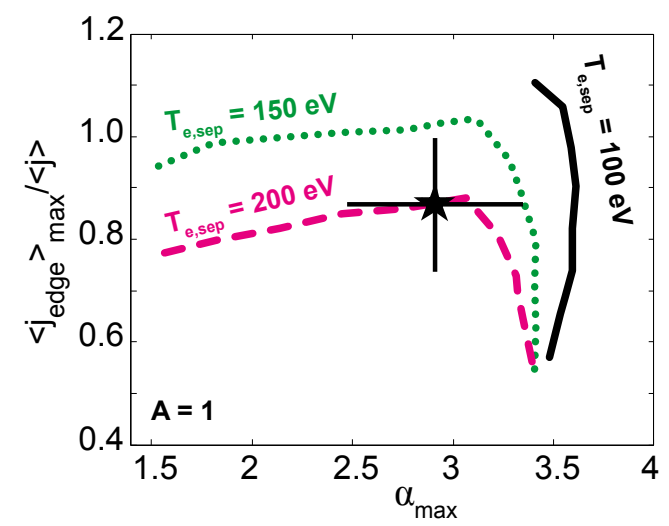

Figure 26. Effect of $T_{\mathrm{e}, \text { sep }}$ on P-B stability of the pre-ELM pedestal of discharge \#91554 as calculated with HELENA/ELITE using the stability criterion $\gamma_{\mathrm{MHD}}>$ $0.25 \times \omega_{\text {dia }}$. The black star indicates the operational point of the pedestal for \#91554. The stability boundary is shown assuming $T_{\mathrm{e}, \mathrm{sep}}=100 \mathrm{eV}$ (solid black), $T_{\mathrm{e}, \mathrm{sep}}=150 \mathrm{eV}$ (dotted green) and $T_{\mathrm{e}, \mathrm{sep}}=200 \mathrm{eV}$ (dashed magenta).

penetration may break down in the investigated plasmas. Interpretative EDGE2DEIRENE simulations required higher anomalous perpendicular transport coefficients $\left(D_{\perp}\right.$ and $\left.\chi_{\perp}\right)$ in $\mathrm{H}$ to match the experimental profiles, indicating that the higher transport in $\mathrm{H}$ than in $\mathrm{D}$ is the main reason for the different pedestals and not neutral penetration.

The inter-ELM separatrix loss power is higher in $\mathrm{H}$ than in $\mathrm{D}$ at similar pedestal top pressure, similar to ASDEX-U [10]. For the isotope dataset, the ELM losses are dominated by particle losses both in $\mathrm{H}$ and D plasmas. At low ELM frequencies, the ELM particle loss increases with increasing $f_{\mathrm{ELM}}$, in correlation with decreasing pedestal top density. Thus, the typically higher ELM frequency in $\mathrm{H}$ than in $\mathrm{D}$ at the same input power and gas rate possibly contributes to density pump out leading to low pedestal density in $\mathrm{H}$.

Pedestal linear MHD stability has been investigated in $\mathrm{H}$ and $\mathrm{D}$, showing that P-B modes are more unstable at lower isotope mass. The direct isotope dependence of linear MHD pedestal stability becomes apparent when the diamagnetic frequency $\left(\omega_{\text {dia }}\right)$ is included in the stability criterion to account for its stabilisation effect. P-B modes are more stable in $\mathrm{D}$ than in $\mathrm{H}$, but the effect is small and alone does not explain the higher pedestal pressure observed in D. Interpretative EDGE2D-EIRENE simulations indicate that the electron temperature at the separatrix could be higher in $H$ than in $\mathrm{D}$ in a pair of type I ELMy H-modes with similar stored energy achieved at twice the power in $\mathrm{H}$, at low gas rate. The largest difference in boundary conditions at the separatrix between $\mathrm{H}$ and $\mathrm{D}$ translates into significant destabilisation of $\mathrm{P}-\mathrm{B}$ modes for the $\mathrm{H}$ pedestal compared to D. This effect is qualitatively consistent with type I ELMs being triggered at lower pedestal densities in the $\mathrm{H}$ case, although the $T_{\mathrm{e} \text {,sep }}$ value in the sensitivity test was chosen as an extreme case and should not be considered at face value. The physics mechanism underlying the profile changes at the plasma edge when the isotope mass is 
varied is not yet understood. Note that direct measurement of the separatrix location would be needed in order to confirm or disprove the higher indicated $T_{\mathrm{e}, \text { sep }}$ in $\mathrm{H}$ than in D.

Results presented in this paper indicate that ELM and inter-ELM transport and pedestal stability could possibly be affected by the isotope mass, leading to a favourable isotope dependence. Further studies are required to be able to provide quantitative predictions beyond the qualitative findings of this work. Changes in pedestal transport in $\mathrm{T}$ and DT plasmas with respect to $\mathrm{H}$ and $\mathrm{D}$ are expected to play an important role in the pedestal and global confinement. The indirect isotope dependence on pedestal MHD stability through the separatrix temperature is not considered to be dominant in $\mathrm{T}$ and DT, because at the higher densities expected in $\mathrm{T}$ and DT (compared to $\mathrm{H}$ and D) the divertor is likely to be in a conduction limited divertor regime with similar target and upstream temperatures as found in EDGE2D-EIRENE for the D case.

Future work includes further studies with the EDGE2D-EIRENE code to examine the role of the particle source and transport in setting the density pedestal. These will also investigate the relative importance of ELM and inter-ELM particle losses by utilising time dependent simulations with simplified ELM models. Further experiments are planned in the upcoming JET campaigns to examine the ELM particle losses in different isotopes by applying ELM trigger techniques to match the ELM frequency in H, D and T plasmas. Experimental analysis and interpretative edge transport analysis suggest that the pedestal transport is likely to play an important role in setting the pedestal height and shape and may be the primary difference between $\mathrm{H}$ and $\mathrm{D}$ pedestals. Thus, future work should also focus on studying the turbulence driving the inter-ELM pedestal transport with gyrokinetic simulations.

\section{Acknowledgments}

The first author would like to thank M. Groth for fruitful discussions. This work was supported by the Engineering and Physical Sciences Research Council [EP/L01663X/1]. This work has been carried out within the framework of the EUROfusion Consortium and has received funding from the Euratom research and training programme 2014-2018 and 2019-2020 under grant agreement No 633053. The views and opinions expressed herein do not necessarily reflect those of the European Commission.

\section{References}

[1] C.F. Maggi, H. Weisen, J.C. Hillesheim, A. Chankin, E. Delabie, L. Horvath, F. Auriemma, I.S. Carvalho, G. Corrigan, J. Flanagan, L. Garzotti, D. Keeling, D. King, E. Lerche, R. Lorenzini, M. Maslov, S. Menmuir, S. Saarelma, A.C.C. Sips, E.R. Solano, E. Belonohy, F.J. Casson, C. Challis, C. Giroud, V. Parail, C. Silva, M. Valisa, and JET Contributors. Isotope effects on L-H threshold and confinement in tokamak plasmas. Plasma Physics and Controlled Fusion, 60(1):014045, 2017. 
[2] R.J. Groebner, M.A. Mahdavi, A.W. Leonard, T.H. Osborne, G.D. Porter, R.J. Colchin, and L.W. Owen. The role of neutrals in high-mode (H-mode) pedestal formation. Physics of Plasmas, 9(5):2134-2140, 2002.

[3] F. Wagner, G. Becker, K. Behringer, D. Campbell, A. Eberhagen, W. Engelhardt, G. Fussmann, O. Gehre, J. Gernhardt, G.V. Gierke, G. Haas, M. Huang, F. Karger, M. Keilhacker, O. Klüber, M. Kornherr, K. Lackner, G. Lisitano, G.G. Lister, H.M. Mayer, D. Meisel, E.R. Müller, H. Murmann, H. Niedermeyer, W. Poschenrieder, H. Rapp, H. Röhr, F. Schneider, G. Siller, E. Speth, A. Stäbler, K.H. Steuer, G. Venus, O. Vollmer, and Z. Yü. Regime of improved confinement and high beta in neutral-beam-heated divertor discharges of the ASDEX tokamak. Phys. Rev. Lett., 49:1408-1412, 1982.

[4] H. Urano, T. Takizuka, Y. Kamada, N. Oyama, H. Takenaga, and the JT-60 Team. Dimensionless parameter dependence of H-mode pedestal width using hydrogen and deuterium plasmas in JT60U. Nuclear Fusion, 48(4):045008, 2008.

[5] H. Urano, T. Takizuka, T. Fujita, Y. Kamada, T. Nakano, N. Oyama, and the JT-60 Team. Energy confinement of hydrogen and deuterium H-mode plasmas in JT-60U. Nuclear Fusion, 52(11):114021, 2012.

[6] H. Urano, T. Takizuka, N. Aiba, M. Kikuchi, T. Nakano, T. Fujita, N. Oyama, Y. Kamada, N. Hayashi, and the JT-60 Team. Hydrogen isotope effects on ITG scale length, pedestal and confinement in JT-60 H-mode plasmas. Nuclear Fusion, 53(8):083003, 2013.

[7] D.P. Schissel, K.H. Burrell, J.C. DeBoo, R.J. Groebner, A.G. Kellman, N. Ohyabu, T.H. Osborne, M. Shimada, R.T. Snider, R.D. Stambaugh, T.S. Taylor, and DIII-D Research Team. Energy confinement properties of H-mode discharges in the DIII-D tokamak. Nuclear Fusion, 29(2):185197, feb 1989.

[8] ASDEX Team. The H-Mode of ASDEX. Nuclear Fusion, 29(11):1959-2040, 1989.

[9] M. Bessenrodt-Weberpals, F. Wagner, O. Gehre, L. Giannone, J.V. Hofmann, A. Kallenbach, K. McCormick, V. Mertens, H.D. Murmann, F. Ryter, B.D. Scott, G. Siller, F.X. Soldner, A. Stabler, K.H. Steuer, U. Stroth, N. Tsois, H. Verbeek, and H. Zohm. The isotope effect in ASDEX. Nuclear Fusion, 33(8):1205-1238, 1993.

[10] F.M. Laggner, E. Wolfrum, M. Cavedon, F. Mink, M. Bernert, M.G. Dunne, P.A. Schneider, A. Kappatou, G. Birkenmeier, R. Fischer, M. Willensdorfer, F. Aumayr, EUROfusion MST1 Team, and ASDEX Upgrade Team. Pedestal structure and inter-ELM evolution for different main ion species in ASDEX Upgrade. Physics of Plasmas, 24(5):056105, 2017.

[11] J.G. Cordey, B. Balet, D.V. Bartlett, R.V. Budny, J.P. Christiansen, G.D. Conway, L.-G. Eriksson, G.M. Fishpool, C.W. Gowers, J.C.M. de Haas, P.J. Harbour, L.D. Horton, A.C. Howman, J. Jacquinot, W. Kerner, C.G. Lowry, R.D. Monk, P. Nielsen, E. Righi, F.G. Rimini, G. Saibene, R. Sartori, B. Schunke, A.C.C. Sips, R.J. Smith, M.F. Stamp, D.F.H. Start, K. Thomsen, B.J.D. Tubbing, and M.G. von Hellermann. Plasma confinement in JET H mode plasmas with H, D, DT and T isotopes. Nuclear Fusion, 39(3):301, 1999.

[12] M. Maslov, A. Boboc, M. Brix, J. Flanagan, M. Romanelli, C. Price, and JET contributors. Energy and particle confinement in JET H-mode plasma. to be submitted to Nuclear Fusion, 2019.

[13] H. Weisen, C.F. Maggi, L. Horvath, F. Auriemma, T.W. Bache, F.J. Casson, A. Chankin, E. Delabie, C. Giroud, D. King, R. Lorenzini, S. Menmuir, and E. Viezzer. Isotope dependence of confinement in JET Deuterium and Hydrogen plasmas. In 27th IAEA Fusion Energy Conference, Ahmedabad, India, pages EX/P1-4, 2018.

[14] R. Pasqualotto, P. Nielsen, C. Gowers, M. Beurskens, M. Kempenaars, T. Carlstrom, D. Johnson, and JET-EFDA Contributors. High resolution Thomson scattering for Joint European Torus (JET). Review of Scientific Instruments, 75(10):3891-3893, 2004.

[15] A. Boboc, M. Gelfusa, A. Murari, P. Gaudio, and JET-EFDA Contributors. Recent developments of the JET far-infrared interferometer-polarimeter diagnostic. Review of Scientific Instruments, 81(10):10D538, 2010. 
[16] D. Reiter. Progress in two-dimensional plasma edge modelling. Journal of Nuclear Materials, 196-198:80-89, 1992.

[17] R. Simonini, G. Corrigan, G. Radford, J. Spence, and A. Taroni. Models and numerics in the multi-fluid 2-D edge plasma code EDGE2D/U. Contributions to Plasma Physics, 34(2-3):368373, 1994.

[18] S. Wiesen. EDGE2D/EIRENE code interface report. IRC Report, 2006. http://www. eirene. de/e2deir_report_30jun06.pdf.

[19] G.T.A. Huysmans, J.P. Goedbloed, and W. Kerner. Isoparametric bicubic Hermite elements for solution of the Grad-Shafranov equation. In Proceedings of the CP90 Conference on Computational Physics. World Scientific Publishing Co. Singapore, 1991.

[20] H.R. Wilson, P.B. Snyder, G.T.A. Huysmans, and R.L. Miller. Numerical studies of edge localized instabilities in tokamaks. Physics of Plasmas, 9(4):1277-1286, 2002.

[21] P.B. Snyder, H.R. Wilson, J.R. Ferron, L.L. Lao, A.W. Leonard, T.H. Osborne, A.D. Turnbull, D. Mossessian, M. Murakami, and X.Q. Xu. Edge localized modes and the pedestal: A model based on coupled peelingâĂŞballooning modes. Physics of Plasmas, 9(5):2037-2043, 2002.

[22] C.F. Maggi, S. Saarelma, F.J. Casson, C. Challis, E. de la Luna, L. Frassinetti, C. Giroud, E. Joffrin, J. Simpson, M. Beurskens, I. Chapman, J. Hobirk, M. Leyland, P. Lomas, C. Lowry, I. Nunes, F. Rimini, A.C.C. Sips, H. Urano, and JET Contributors. Pedestal confinement and stability in JET-ILW ELMy H-modes. Nuclear Fusion, 55(11), 2015.

[23] C.F. Maggi, L. Frassinetti, L. Horvath, A. Lunniss, S. Saarelma, H. Wilson, J. Flanagan, M. Leyland, I. Lupelli, S. Pamela, H. Urano, L. Garzotti, E. Lerche, I. Nunes, F. Rimini, and JET Contributors. Studies of the pedestal structure and inter-ELM pedestal evolution in JET with the ITER-like wall. Nuclear Fusion, 57(11):116012, 2017.

[24] V.P. Bhatnagar, J. Lingertat, R. Barnsley, P. Breger, J.P. Christiansen, S. Clement, J.G. Cordey, S.J. Davies, J.K. Ehrenberg, L.G. Eriksson, G.M. Fishpool, P.J. Harbour, L.D. Horton, J. Jacquinot, H.J. JÃd'ckel, K. Lawson, C.G. Lowry, C.F. Maggi, G.F. Matthews, R.D. Monk, D.P. OBrien, V.V. Parail, E. Righi, G. Saibene, R. Sartori, B. Schunke, A.C.C. Sips, M.F. Stamp, D.F.H. Start, and K. Thomsen. Edge localized modes and edge pedestal in NBI and ICRF heated H, D and T plasmas in JET. Nuclear Fusion, 39(3):353-367, 1999.

[25] R.J. Groebner and T.H. Osborne. Scaling studies of the high mode pedestal. Physics of Plasmas, 5(5):1800-1806, 1998.

[26] L. Frassinetti, M.N.A. Beurskens, R. Scannell, T.H. Osborne, J. Flanagan, M. Kempenaars, M. Maslov, R. Pasqualotto, M. Walsh, and JET-EFDA Contributors. Spatial resolution of the JET Thomson scattering system. Review of Scientific Instruments, 83(1), 2012.

[27] M.J. Leyland, M.N.A. Beurskens, J.C. Flanagan, L. Frassinetti, K.J. Gibson, M. Kempenaars, M. Maslov, R. Scannell, and JET Contributors. Edge profile analysis of Joint European Torus (JET) Thomson scattering data: Quantifying the systematic error due to edge localised mode synchronisation. Review of Scientific Instruments, 87(1), 2016.

[28] P.B. Snyder, R.J. Groebner, A.W. Leonard, T.H. Osborne, and H.R. Wilson. Development and validation of a predictive model for the pedestal height. Physics of Plasmas, 16(5):056118, 2009.

[29] M.N.A. Beurskens, T.H. Osborne, P.A. Schneider, E. Wolfrum, L. Frassinetti, R. Groebner, P. Lomas, I. Nunes, S. Saarelma, R. Scannell, P.B. Snyder, D. Zarzoso, I. Balboa, B. Bray, M. Brix, J. Flanagan, C. Giroud, E. Giovannozzi, M. Kempenaars, A. Loarte, E. de la Luna, G. Maddison, C.F. Maggi, D. McDonald, R. Pasqualotto, G. Saibene, R. Sartori, Emilia R. Solano, M. Walsh, and L. Zabeo. H-mode pedestal scaling in DIII-D, ASDEX Upgrade, and JET. Physics of Plasmas, 18(5):056120, 2011.

[30] M.J. Leyland, M.N.A. Beurskens, L. Frassinetti, C. Giroud, S. Saarelma, P.B. Snyder, J. Flanagan, S. Jachmich, M. Kempenaars, P. Lomas, G. Maddison, R. Neu, I. Nunes, K.J. Gibson, and JETEFDA Contributors. The H-mode pedestal structure and its role on confinement in JET with a carbon and metal wall. Nuclear Fusion, 55(1):013019, dec 2015. 
[31] L. Frassinetti, M.N.A. Beurskens, S. Saarelma, J.E. Boom, E. Delabie, J. Flanagan, M. Kempenaars, C. Giroud, P. Lomas, L. Meneses, C.S. Maggi, S. Menmuir, I. Nunes, F. Rimini, E. Stefanikova, H. Urano, G. Verdoolaege, and JET Contributors. Global and pedestal confinement and pedestal structure in dimensionless collisionality scans of low-triangularity Hmode plasmas in JET-ILW. Nuclear Fusion, 57(1):016012, 2017.

[32] D.R. Hatch, M. Kotschenreuther, S. Mahajan, P. Valanju, and X. Liu. A gyrokinetic perspective on the JET-ILW pedestal. Nuclear Fusion, 57(3):036020, 2017.

[33] D.R. Hatch, M. Kotschenreuther, S.M. Mahajan, G. Merlo, A.R. Field, C. Giroud, J.C. Hillesheim, C.F. Maggi, C. Perez von Thun, C.M. Roach, S. Saarelma, and JET Contributors. Direct gyrokinetic comparison of pedestal transport in JET with carbon and ITER-like walls. Nuclear Fusion, 59(8):086056, 2019.

[34] L. Frassinetti, M.G. Dunne, U. Sheikh, S. Saarelma, C.M. Roach, E. Stefanikova, C. Maggi, L. Horvath, S. Pamela, E. de la Luna, E. Wolfrum, M. Bernert, P. Blanchard, B. Labit, A. Merle, L. Guimarais, S. Coda, H. Meyer, J.C. Hillesheim, the ASDEX Upgrade Team, JET Contributors, the TCV Team, and the EUROfusion MST1 Team. Role of the pedestal position on the pedestal performance in AUG, JET-ILW and TCV and implications for ITER. Nuclear Fusion, 59(7):076038, 2019.

[35] E. Stefanikova, L. Frassinetti, S. Saarelma, A. Loarte, I. Nunes, L. Garzotti, P. Lomas, F. Rimini, P. Drewelow, U. Kruezi, B. Lomanowski, E. de la Luna, L. Meneses, M. Peterka, B. Viola, C. Giroud, C.F. Maggi, and JET contributors. Effect of the relative shift between the electron density and temperature pedestal position on the pedestal stability in JET-ILW and comparison with JET-C. Nuclear Fusion, 58(5):056010, 2018.

[36] L. Frassinetti, D. Dodt, M.N.A. Beurskens, A. Sirinelli, J.E. Boom, T. Eich, J. Flanagan, C. Giroud, M.S. Jachmich, M. Kempenaars, P. Lomas, G. Maddison, C. Maggi, R. Neu, I. Nunes, C. Perez von Thun, B. Sieglin, M. Stamp, and JET-EFDA Contributors. Effect of nitrogen seeding on the energy losses and on the time scales of the electron temperature and density collapse of type-I ELMs in JET with the ITER-like wall. Nuclear Fusion, 55(2):023007, 2015.

[37] A. Loarte, M. Becoulet, G. Saibene, R. Sartori, D.J. Campbell, T. Eich, A. Herrmann, M. Laux, W. Suttrop, B. Alper, P.J. Lomas, G. Matthews, S. Jachmich, J. Ongena, P. Innocente, and EFDA-JET Workprogramme Collaborators. Characteristics and scaling of energy and particle losses during Type I ELMs in JET H-modes. Plasma Physics and Controlled Fusion, 44(9):1815$1844,2002$.

[38] A. Loarte, G. Saibene, R. Sartori, D. Campbell, M. Becoulet, L. Horton, T. Eich, A. Herrmann., G. Matthews, N. Asakura, A. Chankin, A. Leonard, G. Porter, G. Federici, G. Janeschitz, M. Shimada, and M. Sugihara. Characteristics of type I ELM energy and particle losses in existing devices and their extrapolation to ITER. Plasma Physics and Controlled Fusion, 45(9):1549-1569, 2003.

[39] E. de la Luna, I.T. Chapman, F. Rimini, P.J. Lomas, G. Saibene, F. Koechl, R. Sartori, S. Saarelma, R. Albanese, J. Flanagan, F. Maviglia, V. Parail, A.C.C. Sips, and E.R. Solano and. Understanding the physics of ELM pacing via vertical kicks in JET in view of ITER. Nuclear Fusion, 56(2):026001, 2015.

[40] P.C. Stangeby. The Plasma Boundary of Magnetic Fusion Devices. Series in Plasma Physics and Fluid Dynamics. Taylor \& Francis, 2000.

[41] A. Kallenbach, N. Asakura, A. Kirk, A. Korotkov, M.A. Mahdavi, D. Mossessian, and G.D. Porter. Multi-machine comparisons of h-mode separatrix densities and edge profile behaviour in the ITPA SOL and Divertor Physics Topical Group. Journal of Nuclear Materials, 337-339:381$385,2005$.

[42] J. Simpson, D. Moulton, C. Giroud, M. Groth, and G. Corrigan. Using EDGE2D-EIRENE to simulate the effect of impurity seeding and fueling on the upstream electron separatrix temperature. Nuclear Materials and Energy, 20:100599, 2019. 
[43] S. Saarelma, A. Järvinen, M. Beurskens, C. Challis, L. Frassinetti, C. Giroud, M. Groth, M. Leyland, C.F. Maggi, J. Simpson, and JET Contributors. The effects of impurities and core pressure on pedestal stability in Joint European Torus (JET). Physics of Plasmas, 22(5):056115, 2015.

[44] H. Bufferand, B. Bensiali, J. Bucalossi, G. Ciraolo, P. Genesio, Ph. Ghendrih, Y. Marandet, A. Paredes, F. Schwander, E. Serre, and P. Tamain. Near wall plasma simulation using penalization technique with the transport code SolEdge2D-Eirene. Journal of Nuclear Materials, 438:S445 - S448, 2013. Proceedings of the 20th International Conference on Plasma-Surface Interactions in Controlled Fusion Devices.

[45] H. Bufferand, G. Ciraolo, Y. Marandet, J. Bucalossi, Ph. Ghendrih, J. Gunn, N. Mellet, P. Tamain, R. Leybros, N. Fedorczak, F. Schwander, and E. Serre. Numerical modelling for divertor design of the WEST device with a focus on plasma-wall interactions. Nuclear Fusion, 55(5):053025, 2015 .

[46] H. Weisen, A. Zabolotsky, M. Maslov, M. Beurskens, C. Giroud, D. Mazon, and JET-EFDA contributors. Scaling of density peaking in JET H-modes and implications for ITER. Plasma Physics and Controlled Fusion, 48(5A):A457-A466, 2006.

[47] C. Angioni, E. Fable, M. Greenwald, M. Maslov, A.G. Peeters, H. Takenaga, and H. Weisen. Particle transport in tokamak plasmas, theory and experiment. Plasma Physics and Controlled Fusion, 51(12):124017, 2009.

[48] A. Loarte, M.J. Leyland, J.A. Mier, M.N.A. Beurskens, I. Nunes, V. Parail, P.J. Lomas, G.R. Saibene, R.I.A. Sartori, and L. Frassinetti and. Plasma density and temperature evolution following the H-mode transition at JET and implications for ITER. Nuclear Fusion, 53(8):083031, jul 2013.

[49] C. Day. Basics and applications of cryopumps. In CERN Accelerator School, Vacuum in accelerators, Platja d'Aro, Spain, 16-24 May 2006, pages 241-274, 2006.

[50] C. Day. Chapter 8 - Use of porous materials for cryopumping. In Hari Singh Nalwa, editor, Handbook of Surfaces and Interfaces of Materials, pages 265 - 307. Academic Press, Burlington, 2001.

[51] G.T.A. Huysmans, S.E. Sharapov, A.B. Mikhailovskii, and W. Kerner. Modeling of diamagnetic stabilization of ideal magnetohydrodynamic instabilities associated with the transport barrier. Physics of Plasmas, 8(10):4292-4305, 2001.

[52] P.B. Snyder, R.J. Groebner, J.W. Hughes, T.H. Osborne, M. Beurskens, A.W. Leonard, H.R. Wilson, and X.Q. Xu. A first-principles predictive model of the pedestal height and width: development, testing and ITER optimization with the EPED model. Nuclear Fusion, 51(10):103016, 2011.

[53] O. Sauter, C. Angioni, and Y.R. Lin-Liu. Neoclassical conductivity and bootstrap current formulas for general axisymmetric equilibria and arbitrary collisionality regime. Physics of Plasmas, 6(7):2834-2839, 1999.

[54] O. Sauter, C. Angioni, and Y.R. Lin-Liu. Erratum: Neoclassical conductivity and bootstrap current formulas for general axisymmetric equilibria and arbitrary collisionality regime [Phys. Plasmas 6, 2834 (1999)]. Physics of Plasmas, 9(12):5140-5140, 2002.

[55] R.L. Miller, M.S. Chu, J.M. Greene, Y.R. Lin-Liu, and R.E. Waltz. Noncircular, finite aspect ratio, local equilibrium model. Physics of Plasmas, 5(4):973-978, 1998.

[56] N. Aiba, C. Giroud, M. Honda, E. Delabie, S. Saarelma, L. Frassinetti, I. Lupelli, F.J. Casson, S. Pamela, H. Urano, C.F. Maggi, and JET Contributors. Numerical analysis of ELM stability with rotation and ion diamagnetic drift effects in JET. Nuclear Fusion, 57(12):126001, 2017.

[57] N. Aiba, S. Pamela, M. Honda, H. Urano, C. Giroud, E. Delabie, L. Frassinetti, I. Lupelli, N. Hayashi, G. Huijsmans, JET Contributors, and JT-60SA Research Unit. Analysis of ELM stability with extended MHD models in JET, JT-60U and future JT-60SA tokamak plasmas. Plasma Physics and Controlled Fusion, 60(1):014032, 2017. 
[58] N. Aiba, M. Furukawa, M. Hirota, and S. Tokuda. Destabilization mechanism of edge localized MHD mode by a toroidal rotation in tokamaks. Nuclear Fusion, 50(4):045002, 2010. 